Florida International University FIU Digital Commons

\title{
Characterization of cerebral blood flow response to traumatic brain injury in an animal model using SPECT.
}

Michael Capille

Florida International University

DOI: $10.25148 /$ etd.FI14052564

Follow this and additional works at: https://digitalcommons.fiu.edu/etd

Part of the Biomedical Engineering and Bioengineering Commons

\section{Recommended Citation}

Capille, Michael, "Characterization of cerebral blood flow response to traumatic brain injury in an animal model using SPECT." (2005). FIU Electronic Theses and Dissertations. 2035.

https://digitalcommons.fiu.edu/etd/2035 
Miami, Florida

CHARACTERIZATION OF CEREBRAL BLOOD FLOW RESPONSE TO TRAUMATIC BRAIN INJURY IN AN ANIMAL MODEL USING SPECT

A thesis submitted in partial fulfillment of the requirements for the degree of MASTER OF SCIENCE in

BIOMEDICAL ENGINEERING

by

Michael Capille 
To: Dean Vish Prasad

College of Engineering

This thesis, written by Michael Capille, and entitled Characterization of Cerebral Blood Flow Response to Traumatic Brain Injury in an Animal Model using SPECT, having been approved in respect to style and intellectual content, is referred to you for judgment.

We have read this thesis and recommend that it be approved.

Carlos Brain

Mike Georgiou

Nikolaos Tsoukias

Anthony McGoron, Major Professor

Date of Defense: March 28, 2005

The thesis of Michael Capille is approved.

Dean Vish Prasad College of Engineenng

Dean Douglas Wartzok University Graduate School

Florida International University, 2005 


\section{ACKNOWLEDGMENTS}

Thank you to the entire research team: Dr Michael Georgiou and Pablo Sanchez from the Jackson Memorial Hospital Division of Nuclear Medicine, Department of Radiology, Dr. John Kuluz, Dr. Dansha He and Dr. Marcelo Egea from the University of Miami Division of Critical Care Medicine, Department of Pediatrics, the American Heart Association, Florida Puerto Rico Affiliate, the Florence Bayuk Graduate Fellowship and my advisor, Dr. Anthony McGoron, for making this work possible. 
(C) Copyright 2005 by Michael Capille All rights reserved. 


\section{ABSTRACT OF THE THESIS}

CHARACTERIZATION OF CEREBRAL BLOOD FLOW RESPONSE TO

TRAUMATIC BRAIN INJURY IN AN ANIMAL MODEL USING SPECT

by

Michael Capille

Florida International University, 2005

Miami, Florida

Professor Anthony McGoron, Major Professor

In this study the focal effects of moderate traumatic brain injury (TBI) on cerebral blood flow (CBF) as reported by SPECT cerebral brain perfusion (CBP) imaging in an animal model were investigated by parallelized statistical techniques. Subjects were randomly assigned to either a control group or test group receiving fluid percussion injury directly to the left parietal lobe.

Qualitative SPECT CBP accuracy was assessed against reference radioactive microsphere technique (RMT) regional CBF measurements by map reconstruction, registration and smoothing. Cerebral hypoperfusion in the test group was then identified at the voxel level using a statistical parametric mapping (SPM) model.

Using SPM-SPECT CBP, a significant area of hypoperfusion $(\mathrm{P}<0.01)$ was found as a characteristic response to the TBI. Further study and correlation of this characteristic lesion with long-term outcomes and auxiliary diagnostic modalities is critical to developing more effective emergency treatment guidelines and automated medical imaging processing techniques. 
1 Introduction.

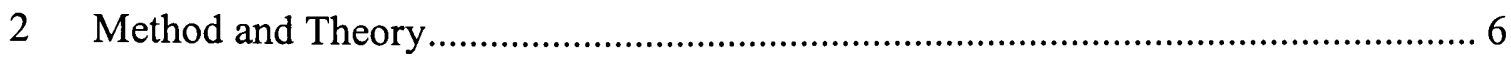

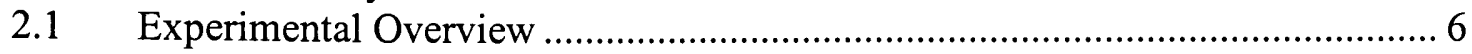

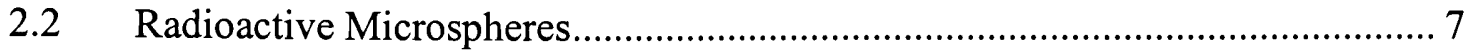

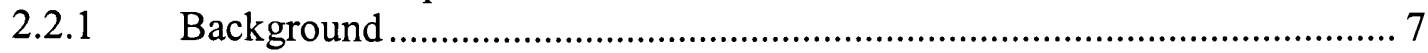

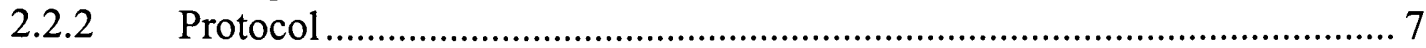

2.3 Ex-Vivo RMT rCBF and In-Vivo SPECT CBP Registration.......................... 10

2.3.1 RMT, Histology and Neuroimaging ...................................................... 10

2.3.2 ROI Maps and Serial Slice Reconstruction .............................................. 11

2.3.3 Microsphere ROI Maps and Serial Slice Registration............................... 12

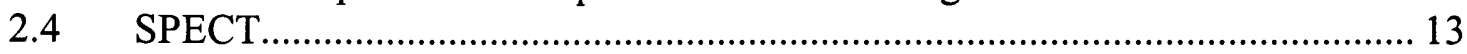

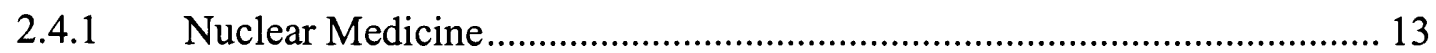

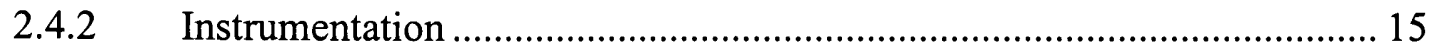

2.5 Image Volume Manipulation and Analysis .................................................... 17

2.6 Statistical Parametric Mapping ........................................................................ 20

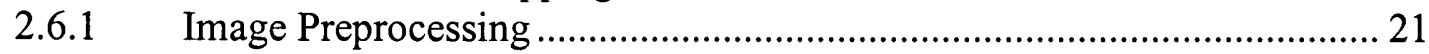

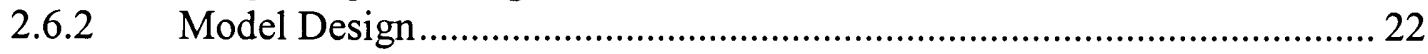

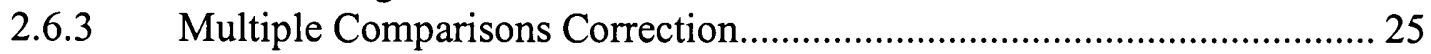

2.7 Verifying SPM Results by ROI Map Analysis .............................................. 26

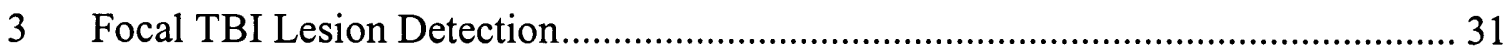

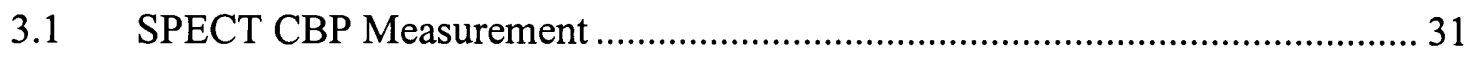

3.1.1 VOI Map Construction from ROI Maps ............................................. 31

3.1.2 VOI Map Registration to SPECT Image Volumes ................................. 34

3.1.3 SPECT CBP Measurement Accuracy ....................................................... 36

3.2 Injury Group Lesion Effect Detection with SPM2 …........................................ 39

3.2.1 Image Preprocessing ........................................................................... 39

3.2.2 Model Specification and Parameter Estimation...................................... 40

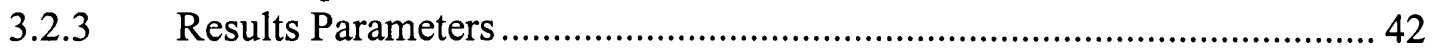

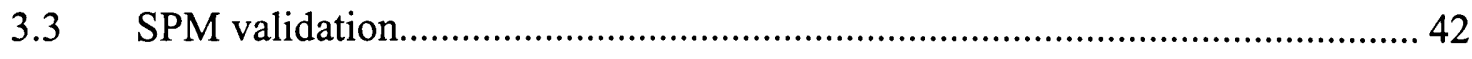

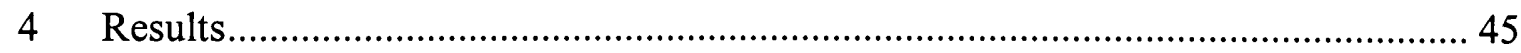

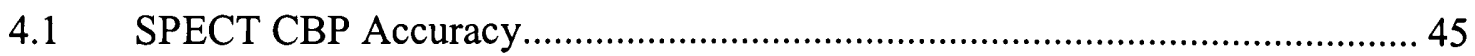

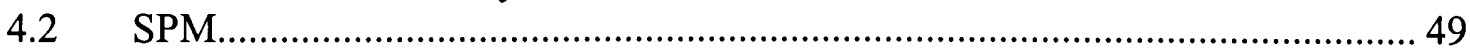

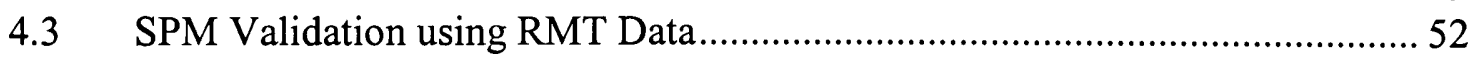

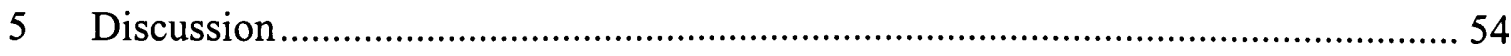

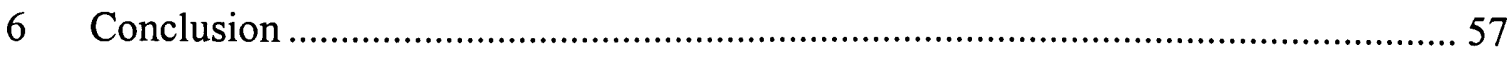


References.

Appendices.. 


\section{LIST OF TABLES}

TABLE

PAGE

Table 1 - Transformation of the ROI slice volume to SPECT volume.

Table 2 - ROI map slices assigned within the SPECT volume space. 33

Table 3 - Linear regression model results for all subjects. . 46

Table 4 - Location and significance values for lesion reported by SPM. 52

Table 5 - RMT rCBF mapping results. 53 


\section{LIST OF FIGURES}

FIGURE

PAGE

Figure 1 - Hierarchical dissection scheme for CBF ROI Map.

Figure 2 - Orthogonal slice view of the piglet head using the PMOD.

Figure 3 - Statistical Parametric Mapping Method. 23

Figure 4 - VOI map of the piglet brain for subject 17. 35

Figure 5 - SPM2 design matrix showing right hemisphere avg CBP predictor 41

Figure 6 - Scatter plot of right hemisphere average rCBF versus rCBF. 44

Figure 7 - Correlation data showing the effect of smoothing of the rCBF data. 47

Figure 8 - Histogram data from subject 10 showing residual regression error. 48

Figure 9 - Histogram data from all subjects showing residual regression error. 48

Figure 10 - Visual validation of spatial registration results with SPM 50

Figure 11 - Significant decreases in SPECT CBP reported by SPM. 51 


\section{LIST OF ACRONYMS}

ANOVA - Analysis of Variance

ANCOVA - Analysis of Covariance

$\mathrm{BP}$ - brain perfusion

BPI - brain perfusion imaging

$\mathrm{ccm}-$ cubic centimeter

CBF - cerebral blood flow

CBP - cerebral blood perfusion

FPI - fluid percussion injury

FWHM - full width half maximum

GRF - Gaussian Random Field

rCBF -regional cerebral blood flow

Resels - resolution elements

RMT - radioactive microspheres technique

ROI - region of interest

SPECT - single photon emission computed tomography

SPM - statistical parametric mapping

TBI - traumatic brain injury

VOI - volume of interest 


\section{Introduction}

Acute traumatic brain injury (TBI) in infants, the leading cause of death and disability in the pediatric population in the United States, is a widespread clinical problem [28]. Effective treatment of TBI critically relies on the early diagnosis of injury severity, however methods for early monitoring and diagnosis remain an active area of research. Advanced diagnostic techniques, such as nuclear medicine imaging, can detect focal decreases in cerebral blood flow (CBF) characteristic of focal lesions resulting from the TBI $[31,32,33]$. Generally, it is proposed that if long-term patient outcomes can be correlated with the appearance of focal decreases in CBF following injury, more effective interventions can be planned leading to more successful patient recovery.

Many nuclear medicine studies designed to assess the correlation between $\mathrm{CBF}$ and severity of TBI are based on Single Photon Emission Computed Tomography (SPECT) cerebral brain perfusion (CBP) imaging [1,2,31]. SPECT is a widely used, non-invasive clinical tool that has proven to be useful not only for TBI but also in additional applications including detection of intracerebral hematomas due to stroke [9] and movement disorders due to closed head injury [10].

This work centers on SPECT CBP based detection of TBI in a pediatric model using piglets and the various computational and statistical methods developed to validate the approach. Localized decreases in regional $\mathrm{CBF}(\mathrm{rCBF})$ have been shown to follow TBI events [8], however not all decreases in rCBF can be detected by SPECT due to 
measurement artifacts such as scatter, photon attenuation and partial volume effects which degrade the source signal. Collective control of these effects often involves design tradeoffs affecting the camera's accuracy and precision. Non-invasive methods for validating SPECT studies are limited. Alternative modalities such as MR [15] and CT [8] reveal anatomical changes (i.e. swelling) of the cortex at the TBI site, however damage at the site is not always accompanied by morphological changes.

In addition to non-invasive methods, invasive methods, such as the radioactive microspheres technique (RMT) are also available. The radioactive microspheres technique is the gold standard for measuring CBF because it does not suffer from the same types of limitations as SPECT imaging, or even non-invasive modalities in general. However, the technique requires that large tissue samples be removed and processed in order to calculate blood flow and is therefore only suitable for animal studies. Both RMT and SPECT methods were carried out simultaneously according to the TBI model protocol. SPECT CBP findings were validated based on measurements derived from RMT.

Consistent with the standard radioactive microsphere protocol, samples are excised from the subject's target tissue under investigation while previously a SPECT camera had taken a "snapshot" of the same target tissue over a large field of view. In order to validate SPECT measurements there must be a method to match, or register, the physical location of the tissue samples removed for microsphere analysis with their corresponding location in the SPECT three-dimensional snapshot [7]. Given the large number of 
microsphere brain tissue samples required by the TBI study protocol, registration for each subject was not performed on a per sample basis but rather on all of the samples in a group with their physical locations and boundaries recorded as a three-dimensional map. Fundamental to the radioactive microsphere technique is the explicit design of a map of tissue areas where blood flow is measured. These areas being studied are referred to as regions of interest (ROIs) with a large number of contiguous ROIs comprising an ROI map. In this work methods were developed to digitize and reconstruct subject specific ROI maps into volume-of-interest (VOI) maps and then perform manual 3-D registration of the digitized VOI maps to the SPECT images.

Accurate registration of the VOI maps allowed both the validation of SPECT data and detection of focal lesions by observing SPECT VOIs with a marked decrease in perfusion. Intrasubject lesion severity was determined by comparing its flow with the homologous region in the contralateral hemisphere of the brain and calculating the percent decrease, similar to the asymmetry index employed by Rousseaux et al. [9].

In order to facilitate intersubject lesion comparison, individual VOIs or aggregated groups of VOIs can be compared. The data reduction and straightforward interpretation of results achieved by these approaches are advantageous. However, focal CBF changes that are either external to the set of predefined groups, obfuscated by a large group, or partially contained by multiple groups can be overlooked. Superior accuracy and precision can be achieved by examining the most basic SPECT image elements: voxels, the three-dimensional analogue of pixels. Voxel level studies, however, require advanced 
computational algorithms to operate on the large data sets and more sophisticated statistical methods to compensate for the massively parallel hypothesis testing.

A voxel, as opposed to VOI, level approach suitable for SPECT CBP image interpretation is statistical parametric mapping (SPM) which is considered part of the broader field of statistical neuroimaging [38]. The SPM methodology has been applied successfully to a range of clinical functional brain studies including head injury $[11,23]$, cognitive rehabilitation therapy [29] and executive brain area functioning after TBI [16]. Detection of focal hypoperfusion requires a statistical model implementation to operate on homologous voxels within and between experimental groups to identify significant differences. Inferences are then made from the test statistics associated with each voxel. In this work, SPM analysis was performed to detect and characterize CBP at the maximum precision afforded by the SPECT machinery and experimental conditions. We hypothesized that voxels in the parietal lobe area of the brain of TBI subjects would show significant hypoperfusion when compared to control subjects.

The standard SPM protocol requires preprocessing of image volumes to provide a sound basis for statistical analysis. First, to facilitate comparison of homologous voxel locations between subjects the image volumes must be anatomically aligned, or coregistered. Second, images must be smoothed in order to overcome intersubject anatomical variation that amplifies experimental error. For the piglet subjects little intersubject variation was seen in the SPECT images because of the relatively small brain size. Lastly, there are concomitant variable effects that must be removed. For example, 
the change in perfusion at a certain voxel may vary from one group to another either due to a valid treatment effect or because the total $\mathrm{CBP}$ was higher, leading to a spurious treatment effect. Concomitant effects are dealt with either by proportional scaling of voxel values across all subjects or ANCOVA with a global measurement of CBP as a predictor variable.

After preprocessing, an appropriate parametric statistical test is developed and applied to the data. Parametric tests (e.g. ANOVA) require that the dependent variable error be normally and independently distributed. However, the independence condition is not readily met because neighboring SPECT voxel values are not independent due to the camera point spread function of the gamma detector and the image reconstruction process. Worsley et al. [12] proposed a correction for these dependence effects based on random field theory. Random field theory constructs an estimate of the independence for each pixel value to adjust its statistical significance. Estimates of independence are based on resels (resolution elements) having the same dimensions as the effective FWHM in each dimension of the reconstructed image. 3D Gaussian random fields recognize the inherent spatial correlation among voxels and provide a means of estimating the number of resels in the image volume. 


\section{Method and Theory}

\subsection{Experimental Overview}

Male piglet subjects weighing $4.1-11.2 \mathrm{~kg}$ were randomly assigned to either a test $(\mathrm{n}=11)$ or control $(\mathrm{n}=8)$ group. The protocol for the care and treatment of the animal subjects was approved by both the Florida International University and University of Miami Institutional Animal Care and Use Committees. All subjects were preanesthetized with an intraperitoneal injection of $40 \mathrm{mg} / \mathrm{kg}$ pentobarbital followed by intravenous administration of $5 \mu \mathrm{g} / \mathrm{kg} / \mathrm{hr}$ fentanyl anesthetic and $0.2 \mathrm{mg} / \mathrm{kg} / \mathrm{hr}$ pancurioum paralytic. Instrumenting of the animal was then performed in order to continuously monitor intracranial pressure, aortic pressure, end tidal respiratory $\mathrm{CO}_{2}$ concentration and body temperature. Lines were provided for injection of radiopharmaceuticals and microspheres, sampling of arterial and venous blood composition and reference organ withdrawal. Both groups received reflection of the scalp, a $1.3 \mathrm{~cm}$ craniotomy over the left parietal cortex and removal of the dura mater. TBI was simulated on test subjects by fluid percussion injury (FPI) at the exposed cortex.

Following injury the animal was transported to the Nuclear Medicine Department at Jackson Memorial Hospital while placed on a ventilator to maintain blood gas and end tidal $\mathrm{CO}_{2}$ concentrations at normal levels. Upon arrival the standard monitoring devices were reconnected. SPECT imaging began approximately 1.5 hours post injury with the "two hour" microsphere injection immediately after. Identical protocols were followed for both control and test subjects with the exception of test subjects receiving TBI. 


\subsection{Radioactive Microspheres}

\subsubsection{Background}

Radioactive microspheres are small $(15 \mu \mathrm{m})$ radiolabeled latex beads that lodge in the capillaries as they pass through the organ tissue [39]. Blood flow in any particular region of tissue is proportional to the radioactivity emitted from the microspheres trapped in the specimen. Calculation of specimen blood flow requires measurements of both the radioactivity per gram of the sample and of the "reference organ", a quantity of arterial blood withdrawn at a known rate. Blood flow in the tissue sample is then calculated as the ratio of tissue sample counts to "reference organ" counts multiplied by the arterial withdrawal (flow) rate.

\subsubsection{Protocol}

The radioactive microsphere technique (RMT) was used in the TBI study to obtain gold standard measurements of $\mathrm{rCBF}(\mathrm{ml} / \mathrm{min} / 100 \mathrm{~g})$. A $100 \mu \mathrm{Ci}$ microsphere solution was injected before injury (real and sham) and immediately after SPECT.

Withdrawal was performed for $1.5 \mathrm{~min}$ at a rate of $1.9 \mathrm{~mL} / \mathrm{min}$ from the arterial line by a syringe pump (Harvard Apparatus, Holliston, MA).

Approximately five hours after injury the subject was euthanized with an intravenous injection of $\mathrm{KCL}(4 \mathrm{~m} \mathrm{Eq} / \mathrm{kg})$. The brain was removed and preserved in 
formalin allowing for the decay of the ${ }^{99 \mathrm{~m}} \mathrm{Tc}-\mathrm{ECD}$ radiopharmaceutical used during the SPECT study. The two nuclides employed in any particular experiment were chosen from a set of possible nuclides including Cerium, Scandium, Niobium and Strontium.

A hierarchical dissection scheme, similar to schemes by Gross et al [40], for the brain is shown in Figure (1). Generally, the dissection scheme can be though of as a brain mapping template for each subject and will be referred to here as either the ROI template map, or template map. The ROI template map defined the number of serial slices and a series of samples contained within each slice. Two important features of the template map were 1) minimal ROI size in order to detect sharp changes in $\mathrm{rCBF}$ and 2) ROI symmetry from the left to right hemisphere facilitating comparison of the injury site to the homologous region on the contralateral side. The ROI size was designed so as not to compromise the integrity of the microsphere measurements. A large number of microspheres per sample were consistently employed in order to achieve minimal standard deviation as suggested by Buckberg [35]. 


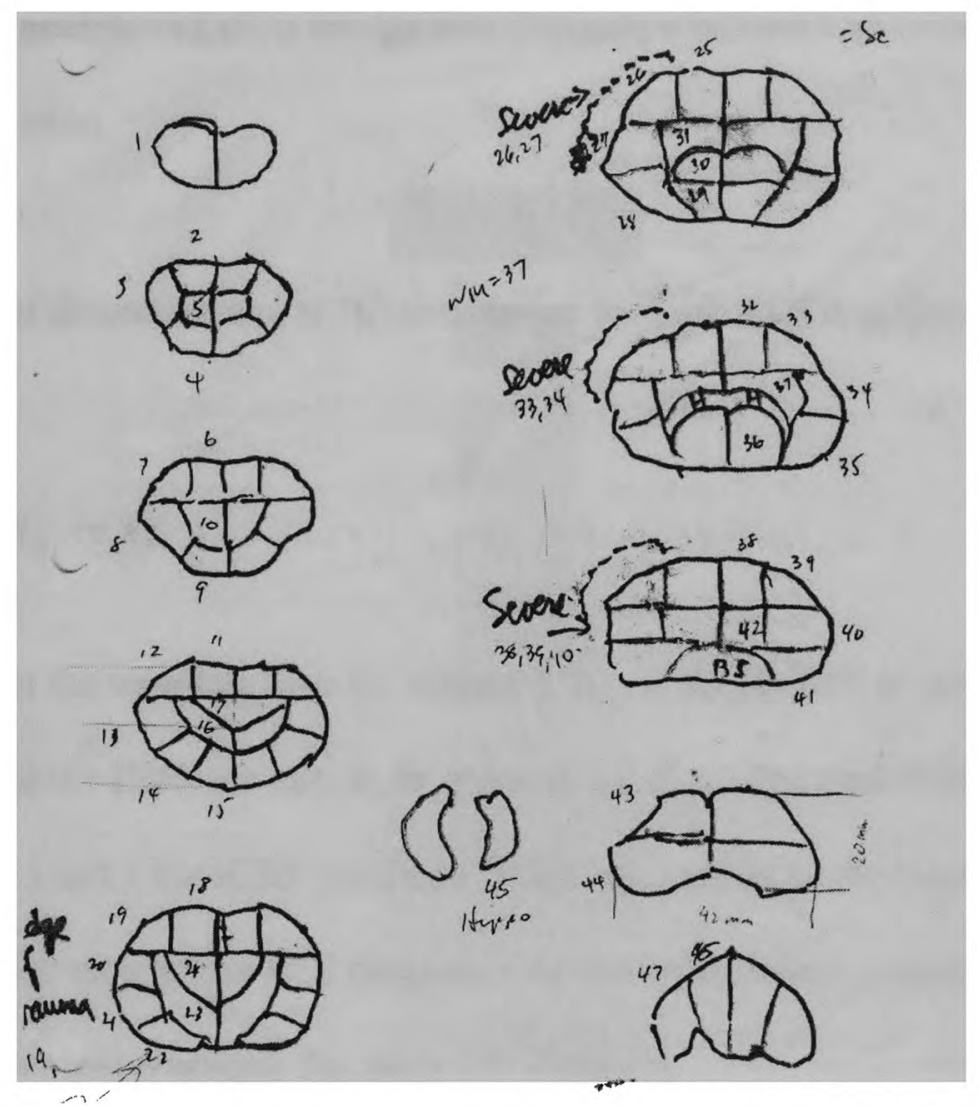

Figure 1 -Hierarchical dissection scheme example displayed as an CBF ROI Map. The specific map for subject $\mathbf{1 0}$ is shown dissected according to the ROI template consisting of nine slices with $\mathbf{8 8}$ ROIs. Each slice is cut symmetrically left to right with the total number of ROIs varying from 2 14. The hippocampus (45) and cerebellum $(46,47)$ are also shown but were not included in the analysis.

Dissection was performed manually, guided by the ROI template map resulting in a subject specific ROI map. Serial slices were made perpendicular to the longitudinal fissure and resulted in an average slice thickness of between 6 and $7 \mathrm{~mm}$. Slices were then placed on heavy weight loose-leaf paper and further cut into symmetrical, left-right, samples following the map specification. After cutting, the outline of each sample was traced while in its natural position within the serial section. Finally, each sample was weighed and its radioactivity (counts) measured by a gamma-well counter (Packard, 
Minaxi Autogamma 5000) within six energy windows, one window for each nuclide.

Spillover of the nuclide radiation energy into alternate windows was corrected by using Gaussian elimination.

Results of dissection and rCBF calculation for each ROI was formally represented as

$$
\Theta_{k}=\left\{R_{j(i)}(v, x)\right\}
$$

where $\theta_{k}$ denotes the template map for subject $k, R_{j(i)}$ is the $j$ th ROI in the $i$ th slice while $j(i)$ expresses that the ROIs are nested, or ordered, by slice. For each ROI $v$ is the volume of the tissue $(\mathrm{mL})$ and $x$ the $\mathrm{rCBF}(\mathrm{mL} / \mathrm{min} / 100 \mathrm{~g})$. In contrast to the hierarchical notation, a simpler view of the ROI template map for each subject ignores nesting of ROIs within slices and reassigns the index $i$ to ROIs (2).

$$
\Theta_{k}=\left\{R_{i}(v, x)\right\}
$$

\subsection{Ex-Vivo RMT rCBF and In-Vivo SPECT CBP Registration}

\subsubsection{RMT, Histology and Neuroimaging}

Reconstruction of ROI maps based on microsphere tissue samples can be thought of as a member of a larger set of methods collectively known as neuroimaging techniques. Histological sampling and autoradiography are widely used neuroimaging methods used to facilitate the comparison of anatomical and functional data $[22,41]$. 
Microsphere brain samples, similar to histological samples, are obtained by first fixing the specimen in tissue protecting alginate embedding material followed by slicing serial sections using a microtome. The number of slices created varies widely depending on the nature of the study being performed. Histological, anatomical mapping and autoradiography studies generally use a highly precise, automated cryotome to achieve a very fine slice thickness in the range of tens of micrometers. Microsphere blood flow studies use a considerably larger slice thickness in the range of millimeters.

\subsubsection{ROI Maps and Serial Slice Reconstruction}

Serial slices are required in order to measure functional data provided by examining each slice, or specific regions in each slice. However, even when not specifically created for anatomical mapping they can provide potentially useful anatomical information to help visualize or analyze functional data $[5,13]$. Unfortunately, the serial sectioning process causes a loss of anatomical information that can negatively affect slice reconstruction. As serial slices are separated from the specimen, they may become warped and no longer represent their in situ morphology. This warping is additional to any changes in morphology introduced by removing the organ from the body.

Also, in the case of coarse serial sectioning, a fair amount of anatomical data can be lost perpendicular to the horizontal axis. Successful reconstruction requires that the serial slices be realigned and, in response, sophisticated block matching algorithms have 
been proposed to realign large numbers of serial sections [20]. Automated algorithms avoid the onerous task of manually realigning many, perhaps thousands, of slices. However, not all neuroimaging methods utilize such a large number of slices. Microsphere methods typically result in a relatively small number of slices whose manual realignment does not present such an onerous task. In this study, only nine slices were generated for each subject. Although the amount of realignment required was minimal, the small number of slices caused a loss of alignment information along the long axis of the brain.

\subsubsection{Microsphere ROI Maps and Serial Slice Registration}

Typically serial slice realignment is performed in order to apply histology or microsphere data to PET, SPECT or MRI image volumes. With such a large array of possible source and target imaging modalities, registration techniques are numerous. Matsumura et al. registered PET and ex vivo autoradiography data using manual matching of ROIs between modalities [42]. Rubins et al. registered VOIs marked on digitized serial slice images and PET data using a maximization of mutual information metric between image pairs [43]. Sorensen et al registered serial slices to MRI by manipulating the position of the brain during sectioning [44].

Various methods can be used to register a set of serial slices, $\theta$, to their homologous slices in a target image volume. Manual methods rely on two distinct kinds of operations performed by the operator. At a high level the operator must identify 
salient features in the target data set to be used for registration (e.g. the location of cerebral boundaries within the head). Head regions where the radiopharmaceutical accumulates can be used as auxiliary markers to identify non-cerebral regions. To complete the registration the operator must then work at a lower level to choose the best fit between slices of the two modalities (e.g. stepping the edges of the slices in both modalities until they overlap). It is common that edges may not perfectly register during visual inspection. In this case, the best fit maximizes the edge registration simultaneously in all dimensions.

In this study, registration of ROI map slices to target SPECT volume slices was achieved using visually guided manual placement. The SPECT volume was manipulated in 3D space until a valid intermodality match was found for all slices in all dimensions. The piglet cerebrum was clearly located in the context of the head profile and reference anatomy points observable in the CBP image. Maximal fit of the entire ROI map, in all three dimensions, was often achieved at the expense of less than maximal positioning of the slices for any particular dimension.

\subsection{SPECT}

\subsubsection{Nuclear Medicine}

Nuclear medicine studies require the use of a scintillation detector positioned at fixed locations outside the body to measure radioactivity released by radioactive compounds, or radiopharmaceuticals, injected into the body. SPECT nuclear imaging 
combined with the ${ }^{99 \mathrm{~m}} \mathrm{Tc}$ labeling of various specific biological compounds is a popular clinical technique to perform blood flow studies of the heart and, most relevant to this study, the brain as well as other major organs or tissues [25]. Radiopharmaceutical compounds, such as ${ }^{99 \mathrm{~m}} \mathrm{Tc}-\mathrm{ECD}$, consist of both radioactive $\left({ }^{99 \mathrm{~m}} \mathrm{Tc}\right)$ and nonradioactive (ECD) components. Radioactive decay of ${ }^{99 \mathrm{~m}} \mathrm{Tc}$ is characterized by the release of $\gamma$ rays from the Technetium nuclei as they transition from an unstable energy state to a steady ground state, a phenomenon referred to as isomeric transition. These $\gamma$ rays, or photons, have a relatively narrow energy spectrum with a sharp peak around $140 \mathrm{KeV}$. When high-energy photons strike the camera NaI scintillation crystal detectors, the crystals emit light that is amplified by an adjacent photomultiplier tubes. Electrical signals generated by the photomultiplier tubes ideally represent one radiation event from the radiopharmaceutical inside the body and is assigned to a position on a $2 \mathrm{D}$ projection plane transaxially perpendicular to the subject. The detectors rotate around the patient in a "step and shoot" manner, creating a set of projection planes at various angles [37].

In order to analyze the results of a nuclear medicine study a set of $2 \mathrm{D}$ projections are reconstructed into a $3 \mathrm{D}$ image volume using mathematical techniques such as filtered back projection and ordered subsets expectation maximization (OS-EM). The structure of an image volume represents the distribution of radioactivity in a way that is easy and natural to understand, stacked 2D cross sectional images of the subject as positioned between the detectors. Volumes are displayed in Cartesian coordinates $(x, y, z)$ in units of millimeters (mm). Formally, a volume can be thought of as a 3D matrix with each 
element containing regularly spaced samples of radioactivity, or counts. Each element also has a standard height, width and depth.

A 2D element having width and height is considered a pixel and is referred to as a voxel when taking on depth. All elements within an image volume have width, height and depth and are therefore voxels. Image volumes are typically isometric cubes, divided into an equal number of isometric voxels.

Discretization of the image as it is captured by the camera leads to a significant partial volume effect. As a result, the intensity value of each voxel does not represent the true intensity value of the location in the subject but rather an average of the voxel with the neighboring voxels. The magnitude of the distortion is dependent on the voxel size of the volume. Higher resolution volumes have a smaller voxel size and less distortion. Effective correction for partial volume effects in SPECT and PET requires knowledge of the boundaries of the gray and white matter of the brain. Often this information is provided by a complementary MRI scan of the subject [36]. In quantitative SPECT studies partial volume correction is critical. For semi-quantitative studies, such as this one, much valuable information can be gained without performing the corrections.

\subsubsection{Instrumentation}

The SPECT camera used in this study is a Philips-ADAC Vertex Plus Hybrid PET/SPECT (ADAC Laboratories, Milpitas, CA) with two $180^{\circ}$ opposed rotating 
detectors with low energy high-resolution collimators and a 5/8" NaI scintillation crystal. Hybrid systems are unique in that they are capable of operating as two fundamentally distinct modalities, SPECT and PET. However, as is customary with hybrid designs, an attempt to meet two opposing sets of criteria leads to tradeoffs. A compromise between resolution and efficiency for the hybrid camera is determined by the thickness of the scintillation crystal. Quality Control (QC) tests were appropriately performed on the camera as required by relevant Departmental and State regulations. For example, Uniformity tests were performed daily and Center of Rotation tests were performed weekly. The software and hardware for image reconstruction was a Philips-ADAC Pegasys (Sun OS) processing workstation for reconstruction of the SPECT image volumes.

Approximately two hours after TBI, before injection of post-injury microspheres, brain blood perfusion SPECT imaging was performed using ${ }^{99 m}$ Tc-ECD. Scanning was initiated approximately ten minutes after $5 \mathrm{mCi}$ intravenous injection of the radiopharmaceutical. Projections were collected over 24 minutes with each detector rotating $180^{\circ}$ in an elliptical orbit with the piglet placed in the supine position. Image volumes were reconstructed using the Philips-ADAC proprietary filtered backprojection algorithm with axial smoothing and a butterworth reconstruction filter with an order of five and cutoff of 0.45 . Attenuation correction was not available. Image volumes consisted of $64 \times 64 \times 64$ voxels each measuring $4.38 \mathrm{~mm}^{3}$. The detector distance ranged from $14 \mathrm{~cm}$ to $20 \mathrm{~cm}$ during rotation resulting in a FWHM of approximately $12 \mathrm{~mm}$ [26]. 


\subsection{Image Volume Manipulation and Analysis}

Medical images begin as raw planar image projection volumes that are reconstructed into image volumes. Because planar projections are only two dimensional, direct examination provides quite limited information. Reconstructed image volumes are used both in research and clinical practice to directly study the distribution of the radiopharmaceutical in the body or target organ.

Many software packages, both freely distributable and license based, are available for viewing and manipulating image volumes. Free packages, such as Osiris (University Hospital of Geneva, Geneva, Switzerland) are relatively feature rich but lack user support, intuitiveness and many sophisticated features. ROI map reconstruction and registration, as detailed in the proceeding sections, required more powerful and unique tools typically found only in licensed software. For these reasons the PMOD (PMOD Technologies, Ltd., Zurich, Switzerland) medical imaging software, developed specifically for nuclear medicine studies $[17,21]$ was chosen for use here.

Requirements for image volume viewing and manipulation and registration include volume loading, navigation and transformation. PMOD supports image volumes stored in various file formats such as Analyze, ECAT, HIDAC, DICOM and RAW as well as graphic formats such as jpeg and specialized formats such as Matlab. Viewing (PGATE) and VOI analysis (PVOI) tools are distinct modules operating under the main PMOD application. The PGATE viewport provided volume navigation simultaneously through the coronal, sagittal and transverse planes, or views. This method for displaying the data, 
orthogonal view, is essential for analyzing image volume data and is standard clinical practice. Orthogonal navigation works by first choosing a 2D location in one plane (e.g. transverse) and then displaying the orthogonal planes in the remaining views (e.g. coronal and sagittal).

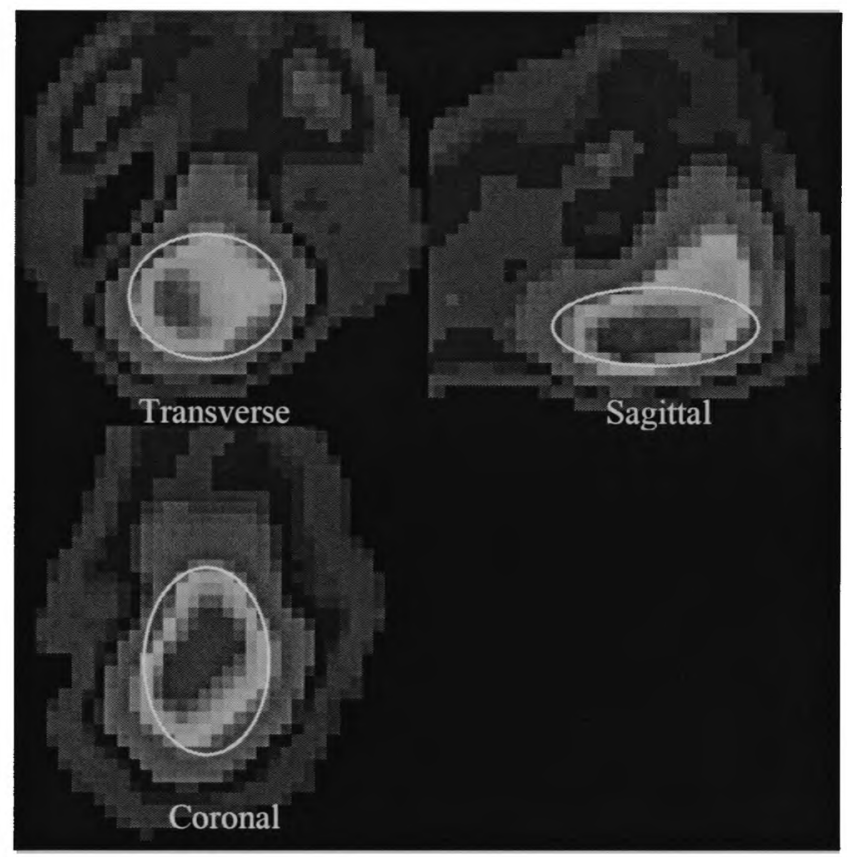

Figure 2 - Orthogonal slice view of the piglet head using the PMOD 3-D Cursor. Each quadrant above represents a 2-D view of the piglet SPECT CBP image volume from each of three interconnected views (transverse, sagittal, coronal). Pixels in each view are color coded according to the magnitude of the counts recorded during acquisition. An approximation of the brain location is given by the ellipses in the three views. On the color intensity scale the brain is represented by the red, yellow and light green areas.

Affine geometric transformations were required to manipulate the image volume for the ROI map registration process. Applied transformations consisted of scaling, rotation and translation in all three dimensions. PMOD applies the scaling transformation first followed by translation. Trilinear interpolation, an extension of linear interpolation into three dimensions, is used when applying transformations. 
Using a one dimensional matrix $A(x)$, linear interpolation predicts a value at $x_{i}$ where $x_{i}$ is not included in the set of $x$ values. $\left.A(x\rfloor\right)$ is the next lowest value of $x$ to $x_{i}$ while $A\left(\lceil x\rceil\right.$ is the next highest value of $x$ to $x_{i} .\lfloor x\rfloor$ denotes the floor of $\mathrm{x}$ while $\lceil x\rceil$ denotes the ceiling. This scheme scales up to three dimensions $A(x, y, z)$ where trilinear interpolation is actually a chain of three linked linear interpolations. Trilinear interpolation is carried out by first calculating the fractional distance between the interpolated point location and the nearest existing locations in each dimension.

$$
\begin{aligned}
& \Delta x=x_{i}-\lfloor x\rfloor \\
& \Delta y=y_{i}-\lfloor y\rfloor \\
& \Delta z=z_{i}-\lfloor z\rfloor
\end{aligned}
$$

Second, perform linear interpolation in the $\mathrm{z}$ dimension

$$
\begin{aligned}
& \left.i_{1}=A(x\rfloor\lfloor y\rfloor\lfloor z\rfloor\right)(1-\Delta z)+A(\lfloor x\rfloor\lfloor y\rfloor\lceil[z\rceil(\Delta z) \\
& i_{2}=A(\lfloor x\rfloor\lceil y\rceil\lfloor z)(1-\Delta z)+A(\lfloor x\rfloor\lceil y\rceil\lceil z\rceil)(\Delta z) \\
& j_{1}=A(\lceil x\rceil\lfloor y\rfloor\lfloor z\rfloor)(1-\Delta z)+A(\lceil x\rceil\lfloor y\rfloor,[z](\Delta z) \\
& j_{2}=A(\lceil x\rceil\lceil y\rfloor\lfloor z\rfloor)(1-\Delta z)+A(\lceil x\rceil\lceil y\rceil,\lceil z\rceil(\Delta z)
\end{aligned}
$$

Third, perform linear interpolation in the y dimension taking into account the results from z. 


$$
\begin{aligned}
& w_{1}=i_{1}(1-\Delta y)+i_{2} \Delta y \\
& w_{2}=j_{2}(1-\Delta y)+j_{2} \Delta y
\end{aligned}
$$

Fourth, perform linear interpolation in the $\mathrm{x}$ dimension taking into account results from $\mathrm{x}$ and y. An interpolated value for $A\left(x_{i}, y_{i}, z_{i}\right)$ is given.

$$
A\left(x_{i}, y_{i}, z_{i}\right)=w_{1}(1-\Delta x)+w_{2} \Delta x
$$

\subsection{Statistical Parametric Mapping}

VOI maps of SPECT perfusion cannot be statistically analyzed as ROI maps due to the highly correlated nature of the measurements between VOIs. In order to analyze the injury groups for significant changes in $\mathrm{rCBF}$ a different strategy must be taken. As in ROI statistical mapping, a univariate test is performed at each data location, which is now an image voxel (counts) rather than an ROI region $\mathrm{rCBF}(\mathrm{mL} / 100 \mathrm{~g} / \mathrm{min})$. Simple correction methods for multiple comparisons between locations assume independence of the counts data at each voxel. As a result the correction is often too severe and statistically significant voxels are lost.

The statistical parametric mapping technique as implemented by SPM2 (Wellcome Department of Imaging Neuroscience, UCL, UK) analyze volumes for group effects at the voxel level and uses statistical theory to derive accurate estimates of data independence. Performing a statistical parametric mapping analysis requires three major 
stages: spatial image transformation, statistical model design, and setting parameters for making inferences on the model output.

\subsubsection{Image Preprocessing}

Each subject image volume must be spatially normalized to a target volume to ensure that the voxel intensity measurements originate from homologous locations in the different brains. Implementing spatial normalization requires considerations for resampling, smoothing, transformation and optimization. Resampling concerns the method of interpolation employed when manipulating image volumes. A range of interpolation options is available including nearest neighbor (simple), trilinear (standard), and sinc (sophisticated). Smoothing is applied to image volumes to overcome difficulties in matching presented by high-frequency information (e.g. sharp edges). Smoothing is computed in the spatial domain using discrete convolution with a Gaussian kernel [4]. The amplitude of a Gaussian at $\mathrm{j}$ units away from the center is defined by:

$$
\begin{aligned}
& g_{j}=\frac{e^{-\frac{j^{2}}{2 s^{2}}}}{\sqrt{2 \pi s^{2}}} \\
& s=\frac{F W H M}{\sqrt{8 \ln (2)}}
\end{aligned}
$$

where $F W H M$ is the full width at half maximum of the Gaussian. A one-dimensional kernel is convolved with an image volume three times, once along each dimension. Affine transformations are a specific type of spatial transformation that constrain 
manipulation of the volume to four operations described by a set of 9 parameters defining varying degrees of translation, rotation, scaling and shearing of the volume in order to normalize brains of different sizes. The transformation origin is the center of image volume. The optimal normalization parameters between a subject and target are determined by minimizing the sum of squared differences between the voxel values of their respective volumes. Normalization as implemented by SPM2 does not require any labels or markers for matching and therefore is completely automated.

\subsubsection{Model Design}

After normalization of the experimental subjects a statistical model can be implemented to analyze the volumes. The general class of statistical models available in SPM can be thought of as subsets of the general linear model framework in equation (3)

$$
Y_{j}=x_{j 1} \beta_{1}+\ldots+x_{j l} \beta_{l}+\ldots+x_{j L} \beta_{L}+\varepsilon_{j}
$$

The variation in $Y_{j}$ is explained in terms of a linear combination of explanatory variables $x_{j l}$, unknown parameters $\beta_{l=l . . L}$, and an error term $\epsilon_{j}$. One of the simpler models that can be derived from the general model is the ANOVA. SPM uses a massively univariate approach that applies an ANOVA test to every voxel in the processed image volumes simultaneously. 


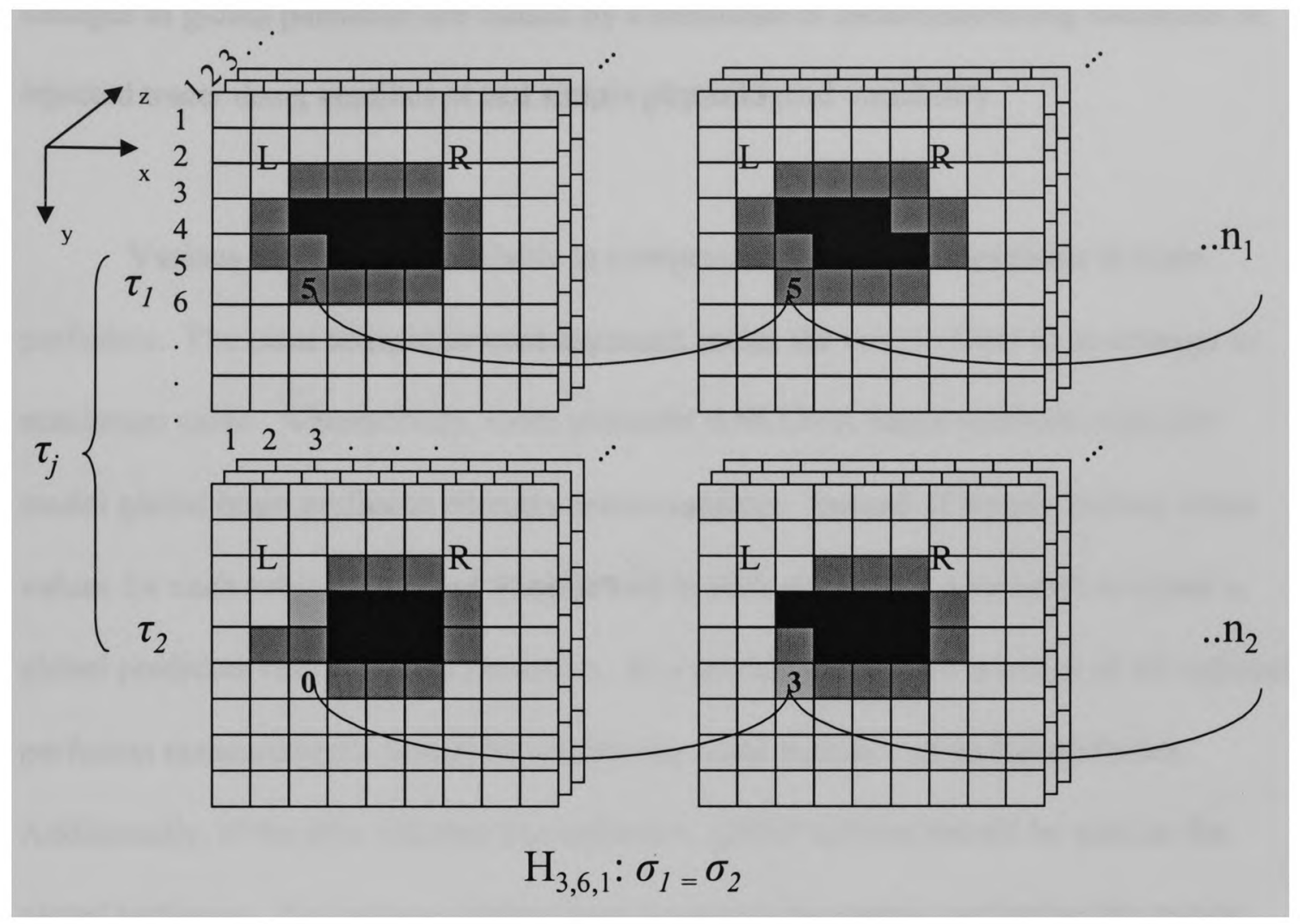

Figure 3 - Statistical Parametric Mapping Method. A statistical model is applied at each voxel in the image independently. For example the null hypothesis, $\mathrm{H}_{0}$, is being tested above with the data point at pixel location $(3,6,1)$ for each subject. The pixel location in the matrix is based on $(x, y, z)$ coordinates with slices stacked in the z-direction. $\tau_{1}$ signifies the first group (i.e. control) containing $n_{1}$ data points $\left\{5,5 \ldots n_{1}\right\}$ with variance $\sigma_{1} . \tau_{2}$ signifies the second group (i.e. test) containing $n_{2}$ data points $\left\{5,5 \ldots n_{2}\right\}$ with variance $\sigma_{2}$. Correction for statistical dependency between voxels due to the smoothness of the image data is performed as a separate step.

Among the most critical design considerations for statistical SPECT models is correcting for differences in global CBF between subjects. The statistical model tests for changes in regional brain perfusion between treatments and therefore regional measurements are likely to be confounded with global brain perfusion fluctuations across subjects. Assuming the same SPECT camera is used for all experimental replications, 
changes in global perfusion are caused by a multitude of factors including variations in injected tracer dose, ventilation and simple physiological variability.

Various methods are available to compensate for subject variations in brain perfusion. The most straightforward approach scales the voxel values to an average or maximum value. Alternatively, more powerful ANCOVA based methods explicitly model global brain perfusion changes across subjects. Instead of simply scaling voxel values for each subject, they are transformed in such a way (e.g. averaged) to create a global predictor value of brain perfusion. In a normal subject, the average of all regional perfusion measurements should be a relatively valid indicator of global perfusion. Additionally, if the area affected was unknown, global activity should be used as the global indicator. If a lesion or deficit were known to be present, including this region would confound the global activity measure. In this case, the global activity should be calculated using areas of the brain known to be unaffected by the treatment. In order to provide the best characterization of the lesion, total perfusion for the right hemisphere represents the variable global activity between subjects.

Once the type of statistical model is chosen, details of the model implementation such as number of experimental treatments (groups), levels of treatments and replications must be specified. This TBI study is classified as a multi-group design. Subjects were randomly assigned to one of two groups, injury or sham, and scanned once. This specific analysis can be carried out using the compare populations (ANCOVA) design in the 
PET/SPECT category of models. ANCOVA is required, as discussed previously, to adjust for subject variations in global brain perfusion.

\subsubsection{Multiple Comparisons Correction}

Applying the univariate statistical model to related voxels across subjects reduces the image volume set to a single volume, or map, of statistic scores (Z-scores). These scores, however, cannot directly be used to test the null hypothesis of equal means between the treatment groups because of the effects of multiple comparisons and spatial correlation between voxels. The problem of multiple comparisons concerns the increasing probability of falsely rejecting the null hypothesis (i.e. false positives) simply due to repeated testing. When multiple comparisons are employed, Bonferroni correction is normally used to adjust the p-values for multiple comparisons of independent tests $[3,27]$. However, in the case of statistical parametric mapping Bonferroni correction becomes too conservative because the voxel scores are not independent due to SPECT smoothing and affine transformations.

In order to control the false positive rate correctly, gaussian random field (GRF) theory estimates the number of independent clusters in an image and combines that information with the Euler characteristic to determine a reliable significance threshold [12]. An accurate measure of the number of independent tests is derived from the number of resels (resolution elements) in an image. Resels are groups of voxels that have dimensions similar to the FWHM of image volume. An image volume with a FWHM of 
$8 \mathrm{~mm}$ in all dimensions and a voxel size of $4 \mathrm{~mm}^{3}$ would have resels consisting of 8 voxels. The expected Euler characteristic is a continuous function jointly determined by the number of resels and the shape of the search area. For a given statistic (e.g. t-statistic) the number of expected false positives is the Euler characteristic. Therefore, a reliable significance threshold can be set where the Euler characteristic equals zero. This corrected threshold is often more liberal than one derived through Bonferroni correction, as would be expected.

\subsection{Verifying SPM Results by ROI Map Analysis}

ROI maps were earlier shown to be a valuable basis for reconstructing VOI maps to analyze SPECT CBP images by considering all ROIs within each subject. In contrast, statistically examining each ROI independently, across all subjects, can provide strong circumstantial evidence of the accuracy of the SPM implementation. Similar to SPM, the result of such processing is an ROI map of $p$-values that provides the most comprehensive view of changes in $\mathrm{rCBF}$ between the control and injury groups. A brief review of the methods basic to statistical ROI mapping is given below. More detailed treatments of the following statistical models can be found in [14]. The simple one-way ANOVA is expressed formally in equation (4).

$$
Y_{i j}=\mu+\tau_{j}+\varepsilon_{i j}
$$


"One-way" refers to one controlled independent variable of interest, or factor, which is to be sampled at different levels $\tau_{j}$ each with $i=1 . . n_{j}$ replications. Each sample value $Y_{i j}$ is a linear combination of the overall mean, $\mu$, plus the difference between the mean of the samples in the $j$ th treatment and the overall mean plus the difference $\epsilon_{i j}$ of the sample value and the treatment mean (5).

$$
Y_{i j} \equiv \mu+\left(\mu_{j}-\mu\right)+\left(Y_{i j}-\mu_{j}\right)
$$

The sample identity (6) is arrived at by drawing samples from each of the populations in (5), making estimates of the grand mean $\bar{Y}_{. .}$and each of the treatments means $\bar{Y}_{. j}$, and moving the first grand mean to the left side.

$$
Y_{i j}-\bar{Y}_{. .}=\bar{Y}_{. j}-\bar{Y}_{. .}+Y_{i j}-\bar{Y}_{. j}
$$

By squaring both sides, summing over $i$ and $j$, and simplifying, (6) becomes the fundamental equation of analysis of variance (7). A dot in place of a variable's subscript index signifies summation of that variable over the replaced index.

$$
\sum_{j=1}^{k} \sum_{i=1}^{n_{j}}\left(Y_{i j}-\bar{Y}_{. .}\right)^{2}=\sum_{j=1}^{k} \sum_{i=1}^{n_{j}}\left(\bar{Y}_{. j}-\bar{Y}_{. .}\right)^{2}+\sum_{j=1}^{k} \sum_{i=1}^{n_{j}}\left(Y_{i j}-\bar{Y}_{. j}\right)^{2}
$$


Equation (7) is often more simply stated as (8)

$$
S S_{\text {total }}=S S_{\text {treatment }}+S S_{\text {error }}
$$

Inferences on the differences of treatment means, $\mu_{j}$, are facilitated by analysis of the treatment and error variances. If there were no treatment effect the error mean square and the treatment mean square (9) would provide equal estimations of the common population variance. Significance is assessed by making inferences based on the F statistic calculated from $\mathrm{MS}_{\text {treatment }} / \mathrm{MS}_{\text {error }}$

$$
\begin{aligned}
& M S_{\text {treatment }}=\frac{S S_{\text {treatment }}}{k-1} \\
& M S_{\text {error }}=\frac{S S_{\text {error }}}{N-k}
\end{aligned}
$$

where $k$ is the last observation and $N$ is the total number of observations. If the $\mathrm{F}$ statistic is large enough for a given set of degrees of freedom the null hypothesis of equal treatment means can be rejected. ANOVA based hypothesis testing assumes that sample error terms $\epsilon_{i j}$ are normally and independently distributed. Inferences should be considered invalid if the departures from normality are strong.

Occasionally when analyzing treatment effects, uncontrolled variables cause additional variability in, or covary with, the dependent variable $Y_{i j}$. Deleterious effects of variables that covary with the dependent variable, also referred to as concomitant 
variables, can be controlled by determining the variance they introduce and subtracting it from the error terms. A standard way to remove concomitant variable effects, in order to properly study a factor of interest, is through analysis of covariance (ANCOVA).

Given a strong linear correlation between the concomitant and dependent variables, a regression model can be expressed similarly to the one-way ANOVA as in (10)

$$
Y_{i j}=\mu+\beta\left(X_{i j}-\bar{X}\right)+\varepsilon_{i j}
$$

where $\beta$ is the true linear regression coefficient between the concomitant variable $X$ and the dependent variable $Y . \bar{X}$ is the mean of the $X$ values. Combining the linear regression (10) and one-way ANOVA (5) gives the complete one-way ANCOVA model (11).

$$
Y_{i j}=\mu+\beta\left(X_{i j}-\bar{X}\right)+\tau_{j}+\varepsilon_{i j}
$$

The reduction in error for $S S_{\text {total }}$ and $S S_{\text {error }}$ is achieved by regression of $\mathrm{Y}$ on the covariate. Ultimately a revised, and more accurate, F statistic is calculated from the adjusted $S S_{\text {total }}$ and $S S_{\text {error }}$.

Statistical ROI mapping requires that ANCOVA be applied, at each ROI in the ROI map, across subjects, in order to determine significant changes in regional activity and 
highlight differences between hemispheres, regions and TBI groups. Carrying out many ANCOVA tests in parallel assumes that the data for each test are independent, or in other words, no random factors exist that affect only a set of data values and not the entire group. The assumption of ROI independence is valid for ROI maps, in stark contrast to the interdependence of voxels in SPECT CBP images.

ANCOVA further assumes that the regression is linear, the slope is not zero, the regression coefficients within each groups are homogeneous and that the concomitant variable is not affected by the treatments given to the groups. 


\section{Focal TBI Lesion Detection}

The fundamental and abstract methods outlined in $(\S 2)$ were implemented to provide a framework for detecting focal TBI by measuring the spatial extent and magnitude of $\mathrm{rCBF}$ and $\mathrm{CBP}$ changes between the control and injury groups. Initially, VOI maps were reconstructed from ROI maps to validate the accuracy of the SPECT CBP studies, demonstrating that CBP changes were correlated with, but not necessarily equal to, the location and magnitude of changes in the gold standard rCBF.

Statistical parametric mapping was implemented to automatically detect focal changes in CBP based on differences between the groups and not a priori ROI definitions. Statistical ROI maps were created apart from SPM to validate the results. Computational tools used to perform the analyses were based on a combination of custom Matlab programming and image manipulation in PMOD. Statistical parametric mapping analysis was implemented through the SPM2 toolbox for Matlab.

\subsection{SPECT CBP Measurement}

\subsubsection{VOI Map Construction from ROI Maps}

ROI map slices were initially contrast enhanced, digitized and stored as jpeg image files. Digitization was performed by an HP Scanjet 3507c and HP Scanning software v1.0. Each serial section was scanned at a resolution of 100 dpi providing for both 
detailed resolution of ROI boundaries and a small file size. Output files were 276 x 276 pixels with 256 gray shades resulting in file sizes less than $100 \mathrm{~KB}$. A total of nine jpeg files were created for each brain, one for each serial slice in the ROI map as in Figure 1.

ROIs from the subject ROI maps, each with volume $v$ and $\operatorname{rCBF} x$, were reconstructed as volume-of-interest (VOI) maps with volume $v$ and counts $y$

$$
R_{i}(v, x) \rightarrow V_{i}(v, y) \quad i=1 . .88
$$

in 3-D space using the biomedical imaging software PMOD v2.5 (PMOD Technologies, Inc). Both the Gateway (PGATE) and VOI (PVOI) modules of PMOD were used extensively during reconstruction for affine image transformation and creation of VOIs, respectively.

Each ROI map slice image was loaded into PGATE as the first slice in a $276 \mathrm{x} 276 \mathrm{x}$ 64 image volume resulting in 63 blank slices plus the ROI map slice image. This loading strategy allowed the slice to be translated and rotated 1) independent of the other ROI map slice images for the same brain and 2) in the same physical space as the SPECT volume. ROI map slice volumes were transformed to the same physical space as SPECT volumes by specifying the voxel dimensions as $1.02 \mathrm{~mm} \times 1.02 \mathrm{~mm} \times 4.38 \mathrm{~mm}$ and the origin coordinates $(0 \mathrm{~mm}, 0 \mathrm{~mm}, 0 \mathrm{~mm})$. 


\begin{tabular}{cccc}
\hline & Dimension & Voxel Size $(\mathrm{mm})$ & Size $(\mathrm{mm})$ \\
\hline $\begin{array}{c}\text { SPECT } \\
\text { Volume }\end{array}$ & $64 \times 64 \times 64$ & $4.38 \times 4.38 \times 4.38$ & $280.32 \times 280.32 \times 280.32$ \\
$\begin{array}{c}\text { ROI Map } \\
\text { Volume }\end{array}$ & $276 \times 276 \times 64$ & $.254 \times .254 \times 4.38$ & $70.10 \times 70.10 \times 280.32$ \\
$\begin{array}{c}\text { Adjusted } \\
\text { ROI Map } \\
\text { Volume }\end{array}$ & $276 \times 276 \times 64$ & $1.02 \times 1.02 \times 4.38$ & $281.52 \times 281.52 \times 280.32$ \\
\hline
\end{tabular}

Table 1 - Transformation of the ROI slice volume space to match the SPECT volume space was accomplished by increasing the reported voxel size. Notice $0.254 \mathrm{~mm} /$ pixel equals $100 \mathrm{dpi}$. Size (mm) is the actual physical size represented by the volume.

After loading, ROI slice image volumes were transformed to a common space. First, a scale factor of 0.37 was applied in the $\mathrm{x}$ and $\mathrm{y}$ dimensions to match the scaling of the SPECT volume. Next the slice was rotated $180^{\circ}$ to match the supine positioning of the animal. Each ROI map slice image was then translated to a pre-specified $\mathrm{z}$ coordinate for that particular slice image as shown in Table 2.

\begin{tabular}{ccc}
\hline $\begin{array}{c}\text { ROI Map } \\
\text { Slice }\end{array}$ & $\begin{array}{c}\text { SPECT Slice } \\
\text { Position }(\mathrm{z})\end{array}$ & $\begin{array}{c}\text { SPECT Physical } \\
\text { Position }(\mathrm{z} \mathrm{mm})\end{array}$ \\
\hline 1 & 27 & -113.88 \\
2 & 25 & -105.12 \\
3 & 23 & -96.36 \\
4 & 21 & -87.60 \\
5 & 19 & -78.84 \\
6 & 17 & -70.08 \\
7 & 15 & -61.32 \\
8 & 13 & -52.56 \\
9 & 11 & -43.80 \\
\hline
\end{tabular}

Table 2 - ROI map slices are assigned within the SPECT volume space. One ROI map slice is assigned to two SPECT slices. For example, ROI map slice 1 occupies SPECT slice position 26 and 27. 
Finally, the midsagittal line of each slice was translated to $136.68 \mathrm{~mm}$ on the $\mathrm{x}$ axis and the superior edge to $-171.36 \mathrm{~mm}$ on the $\mathrm{y}$ axis. Any slices that were not originally drawn with midlines parallel to the $\mathrm{x}$ and $\mathrm{y}$ axes were corrected by rotation about its center.

Following registration of each slice image volume to the common space, the volume was exported to PVOI for further processing. VOI slices were created by manually tracing individual ROI boundaries in the slice image. ROIs taken from the right hemisphere of the brain were traced first in numerical order and the process then repeated for the left hemisphere. After a VOI slice was created it was exported to a VOI definition file for later registration to the SPECT image volume.

\subsubsection{VOI Map Registration to SPECT Image Volumes}

SPECT image volumes were loaded into PGATE for preprocessing with the parameters outlined in Table 1. Volume scale was set to 1.5 in the $\mathrm{x}, \mathrm{y}$ and $\mathrm{z}$ dimensions to adjust for the fractional slice thickness resulting from the brain dissection. ROI map slices were cut with a thickness between $6 \mathrm{~mm}$ and $7 \mathrm{~mm}$, however the SPECT transaxial slice thickness was $4.38 \mathrm{~mm}$. Scaling by 1.5 effectively decreased the SPECT slice thickness to $3.285 \mathrm{~mm}$ allowing assignment of two SPECT transaxial slices to one ROI map serial slice. The scale factor and 2:1 assignment scheme was fixed for all subjects. 


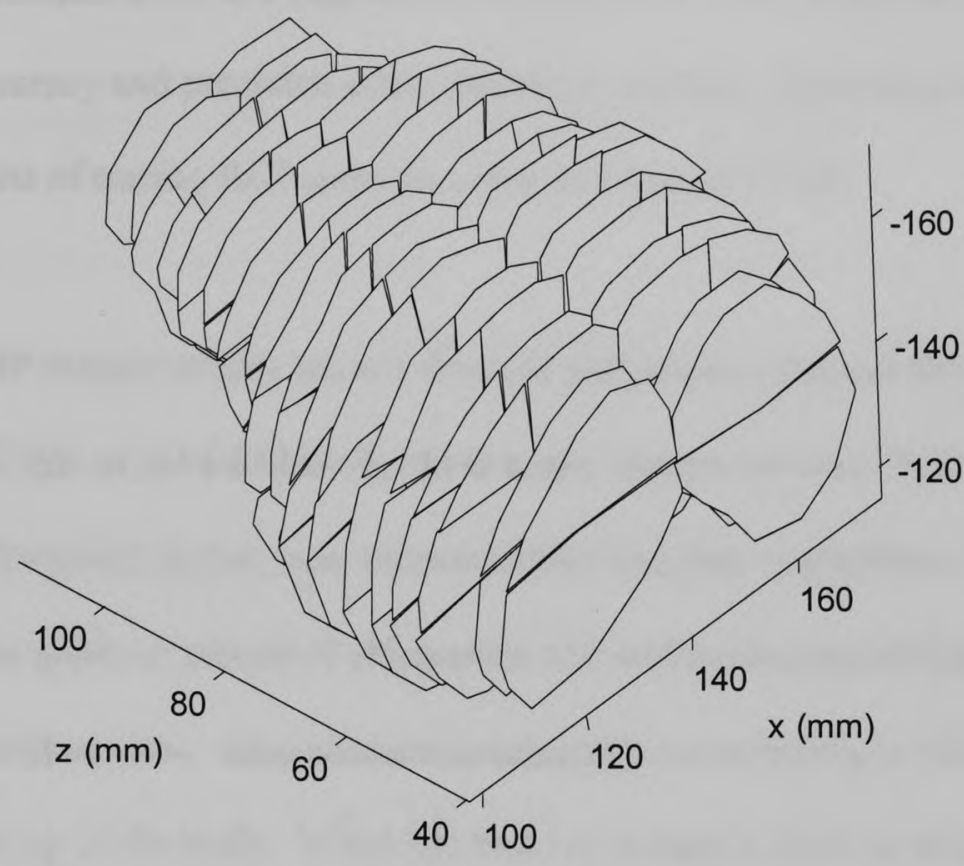

$y(\mathrm{~mm})$

Figure 4 - VOI map of the piglet brain for subject 17 reconstructed from the subject's ROI map. VOI map slices were duplicated to match thickness of scaled SPECT volume.

Following the scale operation, course affine transformations were performed on the image volume to align the subject's brain into the same common space as the reconstructed ROI maps. Complete VOI maps were then imported into PVOI along with the corrected SPECT image volume and the final transformations were made to visually align the VOIs to the contour of the brain in all three dimensions. Averaged counts, sum total counts and VOI volume $(\mathrm{ccm})$ were calculated and stored for each VOI. Counts per gram for each VOI were obtained by dividing the sum total counts by VOI volume and multiplying by the inverse of cortex density $(1.05 \mathrm{~g} / \mathrm{mL})$. 


\subsubsection{SPECT CBP Measurement Accuracy}

Both the reconstruction and registration components of the mapping methodology were tested for accuracy and precision using statistical analysis. Reconstruction tests examined the results of tracing ROI maps on paper and then in PVOI.

SPECT CBP measurements are not absolute indications of blood flow, unlike microsphere $\mathrm{rCBF}$, due to deterioration by three major sources of error: SPECT CBP imaging artifacts discussed earlier, map reconstruction and map registration. Image artifacts include the physical effects of attenuation and scatter and smoothing caused by pixelation of the field of view. Map reconstruction errors occur during excising, dissection, and tracing of the brain. When the brain is excised it tends to widen laterally and shorten vertically although this effect is minimized by the formalin treatment. Map registration errors are introduced by the operator performing registration of the VOI map to the brain in the CBP images. Low quality images, caused by low uptake of the radiopharmaceutical tracer, can cause uncertainty over the correct organ landmarks in the image to align to the VOI map. Also, the VOI map must be aligned at points that are a multiple of the voxel size. For a large voxel size, this also may introduce error if the correct position for the VOI map is thought to be at a position in between two neigboring voxels, or planes.

In examining the accuracy of the SPECT CBP data the imaging, reconstruction and registration errors were considered collectively. The strength of the linear 
relationship between $\mathrm{rCBF}$ and $\mathrm{CBP}$ for each subject was tested using correlation analysis.

\subsubsection{Assessing Accuracy through Linear Regression Analysis}

With the exception of extremely low or high flow values, $\mathrm{rCBF}$ and $\mathrm{CBP}$ are linearly related. Therefore, a suitable model for this relationship is the standard linear model of two variables. A predictive relationship between $\mathrm{rCBF}$ and $\mathrm{CBP}$ was established for each subject by fitting the 88 paired ROI-VOI measurements to a line using linear regression analysis. Both $\mathrm{rCBF}$ and $\mathrm{CBP}$ were normalized using the average value to a range between zero and one to compensate for the different units of measurement $[24,30]$. If the imaging, reconstruction and registration errors were minimal, the linear model would be a good predictor of CBP given $\mathrm{rCBF}$.

Linear regression analysis using the simple straight-line model

$$
C B P_{r C B F}^{\prime}=c_{0}+c_{1} r C B F
$$

computes predicted values of $\mathrm{CBP}, C B P^{\prime}$, given an independent value of $\mathrm{rCBF}$ and slope, $c_{l}$, and intercept, $c_{0}$. Values for $c_{l}$ and $c_{0}$ are derived using the experimental paired data points at each CBP-VOI and rCBF-ROI pair according to the standard least squares normal equations 


$$
\begin{aligned}
& c_{1}=\frac{\sum_{i} \sum_{j} r C B F_{i} C B P_{i j}-\frac{\left(\sum_{i} \sum_{j} r C B F_{i}\right)\left(\sum_{i} \sum_{j} C B P_{i j}\right)}{N}}{\sum_{i} \sum_{j} r C B F_{i}^{2}-\frac{\left(\sum_{i} \sum_{j} x_{j}\right)^{2}}{N}} \\
& c_{0}=\overline{C B P}-c_{1} \overline{r C B F}
\end{aligned}
$$

From these linear regression calculations two important indicators of the $\mathrm{rCBF}$ and $\mathrm{CBP}$ relationship are obtained. A slope, $c_{1}$, of 1 coupled with an intercept, $c_{0}$, of 0 represents perfect agreement between rCBF and CBP values. Normally, these values will be less than optimal.

The correlation coefficient, $r$, also measures the degree to which there is a linear relationship between the two variables and always falls between -1 and 1 . This value does not provide any information as to what the slope may be, only the linearity of the data distribution. A value of 0 signifies no relationship while a value of 1 is considered a perfect positive relationship. A negative $r$ would describe a scenario where one measure of flow is rising while the other falls and would not be considered as valid evidence of the relationship being tested.

Linear models and correlation coefficients were calculated for each subject and the entire population using the regress and corr2 functions in Matlab (Mathworks, Natick, MA). 


\subsection{Injury Group Lesion Effect Detection with SPM2}

In the previous section the ROI Maps of $\mathrm{rCBF}$ values were analyzed for significant changes in rCBF due to TBI. The same analysis cannot be performed on the VOI Maps derived from SPECT images because the CBP measurements are not independent and therefore no simple algorithm is available for adjusting the $\mathrm{p}$ value at each VOI for multiple comparisons. A statistical parametric mapping analysis, through SPM2, was employed to ultimately provide reliable estimates of independence and guidelines for inferring significant effects.

\subsubsection{Image Preprocessing}

Image volumes generated by the hybrid PET/SPECT camera stored in the raw data file format required conversion to the Analyze image file format for importation to SPM2. Subject 17 served as a template, or target, image to which all other subjects were spatially normalized. This particular subject was chosen because it was a control, had high contrast (i.e high counts) and was ideally positioned in the gantry during the scanning process. Also, for optimal results the normalization algorithm requires that the selected template image have very similar contrast to the image from which the parameters are to be determined. 


\subsubsection{Model Specification and Parameter Estimation}

The statistical model for comparing injured and sham groups was implemented as the predefined SPM SPECT model "Compare Populations 1 scan/subject ANCoVA". This model performs voxel-wise unpaired t-tests between the control and test groups while taking the global CBP confounding effect into account using ANCOVA.

$$
\begin{aligned}
& {\left[\begin{array}{l}
Y_{1} \\
Y_{j} \\
Y_{n}
\end{array}\right]=\beta_{1}\left[\begin{array}{l}
x_{11} \\
x_{1 j} \\
x_{1 n}
\end{array}\right]+\beta_{2}\left[\begin{array}{l}
x_{21} \\
x_{2 j} \\
x_{2 n}
\end{array}\right]+\left[\begin{array}{l}
\varepsilon_{1} \\
\varepsilon_{j} \\
\varepsilon_{n}
\end{array}\right]} \\
& \text { or more succinctly } \\
& \underline{Y}=\beta_{1} \underline{X_{1}}+\beta_{2} \underline{X_{2}}+\underline{E} \\
& \mathrm{j}=\text { scan number up to } \mathrm{n} \text { scans } \\
& 1=\text { control group, } 2=\text { test group }
\end{aligned}
$$

The confounding effect, or nuisance variable in SPM2 parlance, is the background level of perfusion (counts/pixel) in the brain. The background level of perfusion was calculated as the right hemisphere average similarly to the ROI map analysis.

$$
X_{r h}=\frac{1}{44} \sum_{i=45}^{88} V_{i}(y)
$$


All normalized images were loaded into SPM2 as belonging to either the test or control group. Each calculated subject covariate was entered as a single nuisance variable.

ANCOVA was selected as the grand mean scaling method and no further grand mean scaling was used. The threshold for determining brain activity was relative and set, relying on general knowledge of the images, to 0.5 . No sphericity correction was applied.

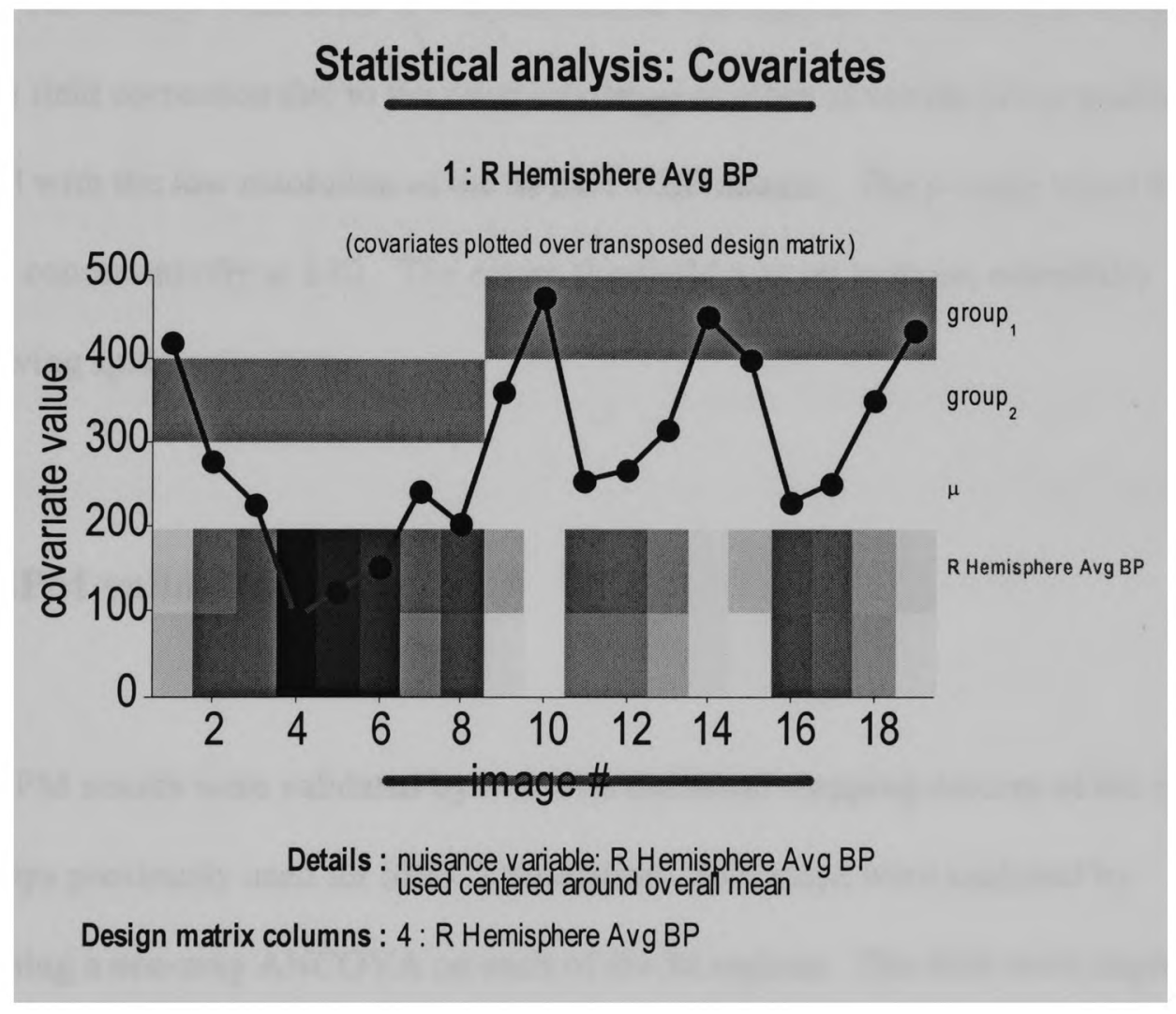

Figure 5 - SPM2 design matrix showing right hemisphere avg CBP predictor plotted for each subject transposed over design matrix. Image number on $\mathbf{x}$-axis corresponds to subject number. Covariate (or nuisance) value on y-axis is right hemisphere avg value (counts). The control group is listed as group $_{1}$ and the test group is listed as group ${ }_{2}$. The axis to the right outlines how the horizontal rows in the design matrix correspond to equation (14). Gray scale is used to visually display numeric values ranging from low (black) to high (white). 


\subsubsection{Results Parameters}

Using the contrast manager GUI a contrast was implemented to specify the effect of interest as any significant decrease in the test group compared to the control group.

$$
\underline{c}_{1}^{T}=(-1,1)
$$

No Family-Wise Error (FWE) correction was applied in addition to the gaussian random field correction due to the relatively small number of voxels being studied coupled with the low resolution of the SPECT CBP images. The p-value voxel threshold was set conservatively at 0.01 . The extent threshold was set to three, essentially disallowing spike activations.

\subsection{SPM validation}

SPM results were validated by a similar statistical mapping process of the rCBF ROI maps previously used for SPECT validation. ROI maps were analyzed by performing a one-way ANCOVA on each of the 88 regions. The tests were implemented using the ANCOVA model previously discussed (11).

$$
Y_{i j}=\mu+\beta\left(X_{i j}-\bar{X}\right)+\tau_{j}+\varepsilon_{i j}
$$


For the ROI map analysis, $i=1 . . n_{j}$, with $n_{j}$ being the number of replications in the $j$ th treatment. The control and injury treatments are represented as $(j=1)$ and $(j=2)$ respectively. Replications for the treatment groups are $n_{l}=8$ and $n_{2}=11$. Each ANCOVA is only concerned with a particular ROI and $Y_{i j}$ is the rCBF value of the $i$ th replication in the $j$ th treatment. The second term is the covariate term that controls for subject specific variation in CBF. The covariate term $\beta\left(X_{i j}-\bar{X}\right)$ is based on average right hemisphere $\mathrm{CBF}$. Controlling for subject specific $\mathrm{CBF}$ ensures that changes in $\mathrm{rCBF}$ can be attributed to any TBI effect that may exist, and not to variations in $\mathrm{CBF}$.

As with the SPM analysis, average right hemisphere CBF, in contrast to global $\mathrm{CBF}$, provides the most accurate measure of a subject's normal CBF given the $a$ priori information that the right side of the brain was not subject to the treatment effect. Estimates of average right hemisphere $\mathrm{CBF}(\mathrm{mL} / \mathrm{min} / 100 \mathrm{~g})$ were calculated for each subject according to (16) and used as the predictor variable for ANCOVA.

$$
X_{r h}=\frac{1}{44} \sum_{i=45}^{88} R_{i}(x)
$$

In Figure 6 the required linear covariance relationship between average right hemisphere $\mathrm{CBF}$ and $\mathrm{rCBF}$ can be seen. 


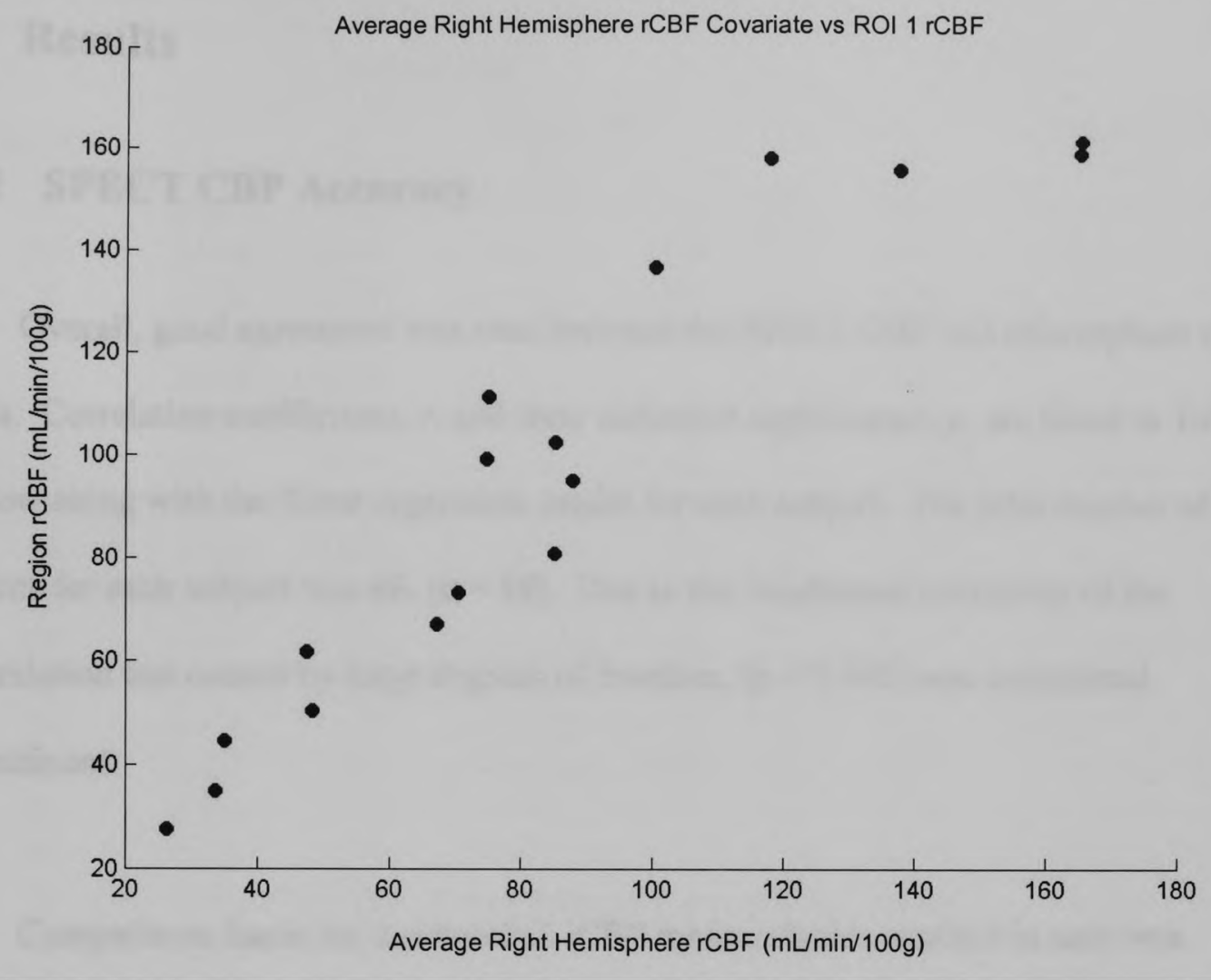

Figure 6 - Scatter plot of right hemisphere average rCBF versus $\mathrm{rCBF}$ for a single ROI. The linear relationship with non zero slope between average $\mathrm{rCBF}$ and the single ROI proves suitability of ANCOVA Covariate.

In order to examine each ROI independently, 88 ANCOVA implementations were performed. Manual entry of the data and collection of test results was not a feasible option given this requirement. To make analysis manageable a toolbox was written in Matlab to automate the analysis. See appendix A for an outline of the data structures and auxiliary functions used to facilitate storing, organizing and manipulating large data sets. 


\section{Results}

\subsection{SPECT CBP Accuracy}

Overall, good agreement was seen between the SPECT CBP and microsphere rCBF data. Correlation coefficients, $r$, and their statistical significance, $p$, are listed in Table 3 below along with the linear regression model for each subject. The total number of data points for each subject was $88,(n=88)$. Due to the heightened sensitivity of the correlation test caused by large degrees of freedom, $(\mathrm{p}<0.001)$ was considered significant.

Comparisons based on unsmoothed $\mathrm{rCBF}$ measurements resulted in only two subjects obtaining high, $(r=0.521)$, and moderate, $(r=0.366)$, correlations and achieving statistical significance at $(p<0.001)$. The lowest correlation coefficients ranged from zero to negative. $\mathrm{CBP}$ and $\mathrm{rCBF}$ should increase or decrease in unison and therefore the negative values were considered just as problematic as those cases reporting a completely random relationship. The mean value for unsmoothed CBP-rCBF correlation, $(0.056 \pm$ 0.210), points to the absence of a linear relationship. Further, visual inspection reveals the relationship is virtually random. Clearly these results demonstrate a large amount of error compounded throughout the various processes culminating in SPECT CBP measurement. 


\begin{tabular}{|c|c|c|c|c|c|c|}
\hline \multirow[b]{2}{*}{ Subject } & \multicolumn{3}{|c|}{ Unsmoothed } & \multicolumn{3}{|c|}{ Smoothed } \\
\hline & $r$ & $p$ & $\begin{array}{c}\text { Regression } \\
\text { Model }\end{array}$ & $r$ & $p$ & $\begin{array}{c}\text { Regression } \\
\text { Model }\end{array}$ \\
\hline 9 & 0.521 & 0.000 & $y=0.294 x+0.706$ & 0.746 & 0.000 & $y=0.464 x+0.536$ \\
\hline 10 & 0.264 & 0.013 & $y=0.234 x+0.766$ & 0.793 & 0.000 & $y=0.819 x+0.181$ \\
\hline 12 & 0.113 & 0.293 & $y=0.057 x+0.943$ & 0.570 & 0.000 & $y=0.370 x+0.630$ \\
\hline 13 & -0.118 & 0.272 & $y=-0.067 x+1.067$ & 0.224 & 0.036 & $y=0.149 x+0.851$ \\
\hline 14 & 0.366 & 0.000 & $y=0.219 x+0.781$ & 0.758 & 0.000 & $y=0.572 x+0.428$ \\
\hline 15 & 0.156 & 0.146 & $y=0.102 x+0.898$ & 0.479 & 0.000 & $y=0.313 x+0.687$ \\
\hline 16 & 0.001 & 0.992 & $y=0.001 x+0.999$ & 0.370 & 0.000 & $y=0.246 x+0.754$ \\
\hline 17 & -0.299 & 0.005 & $y=-0.186 x+1.186$ & 0.181 & 0.091 & $y=0.133 x+0.867$ \\
\hline 18 & -0.289 & 0.006 & $y=-0.133 x+1.133$ & 0.386 & 0.000 & $y=0.234 x+0.766$ \\
\hline 21 & 0.179 & 0.096 & $y=0.093 x+0.907$ & 0.700 & 0.000 & $y=0.420 x+0.580$ \\
\hline 22 & -0.037 & 0.734 & $y=-0.025 x+1.025$ & 0.627 & 0.000 & $y=0.476 x+0.524$ \\
\hline 23 & -0.068 & 0.531 & $y=-0.035 x+1.035$ & 0.650 & 0.000 & $y=0.345 x+0.655$ \\
\hline 25 & 0.118 & 0.273 & $y=0.045 x+0.955$ & 0.555 & 0.000 & $y=0.298 x+0.702$ \\
\hline 26 & 0.021 & 0.849 & $y=0.013 x+0.987$ & 0.523 & 0.000 & $y=0.365 x+0.635$ \\
\hline 28 & 0.098 & 0.366 & $y=0.053 x+0.947$ & 0.345 & 0.001 & $y=0.276 x+0.724$ \\
\hline 29 & -0.017 & 0.873 & $y=-0.011 x+1.011$ & 0.548 & 0.000 & $y=0.470 x+0.530$ \\
\hline 30 & -0.051 & 0.639 & $y=-0.027 x+1.027$ & 0.599 & 0.000 & $y=0.449 x+0.551$ \\
\hline 31 & -0.086 & 0.581 & $y=-0.045 x+1.035$ & 0.680 & 0.000 & $y=0.355 x+0.630$ \\
\hline 32 & 0.005 & 0.990 & $y=0.003 x+0.989$ & 0.573 & 0.000 & $y=0.436 x+0.566$ \\
\hline Overall & 0.007 & 0.002 & $y=0.046 x+0.954$ & 0.285 & 0.000 & $y=0.371 x+0.629$ \\
\hline Corrected & 0.014 & 0.000 & $y=0.067 x+0.933$ & 0.344 & 0.000 & $y=0.404 x+0.596$ \\
\hline
\end{tabular}

Table 3 - Correlation coefficients ( $r$ ), their associated p-values (p) and the computed linear regression for all subjects. Results based on unsmoothed rCBF values are in columns two through four while results based on smoothed rCBF reside in columns five through seven.

Comparing the right side of Table 3 with the left provides clear evidence that the smoothing of the rCBF measurements accounted for a large portion of the experimental error contributed by imaging artifacts and VOI map reconstruction and registration inaccuracies. The success of this smoothing approach can also be found in a similar study of multislice first-pass cardiac perfusion MRI by Epstein et al. [19]. The increase in accuracy is reflected by the higher correlation coefficients and slopes of the linear regression equations. This highly material improvement is demonstrated in the mean $r$ 
$(0.532 \pm 0.181)$, maximum $r(0.793)$, and minimum $r(0.181)$. No negative correlation coefficients survived the smoothing process. An example of the regression model improvement for a subject 10 is shown in Figure 5.

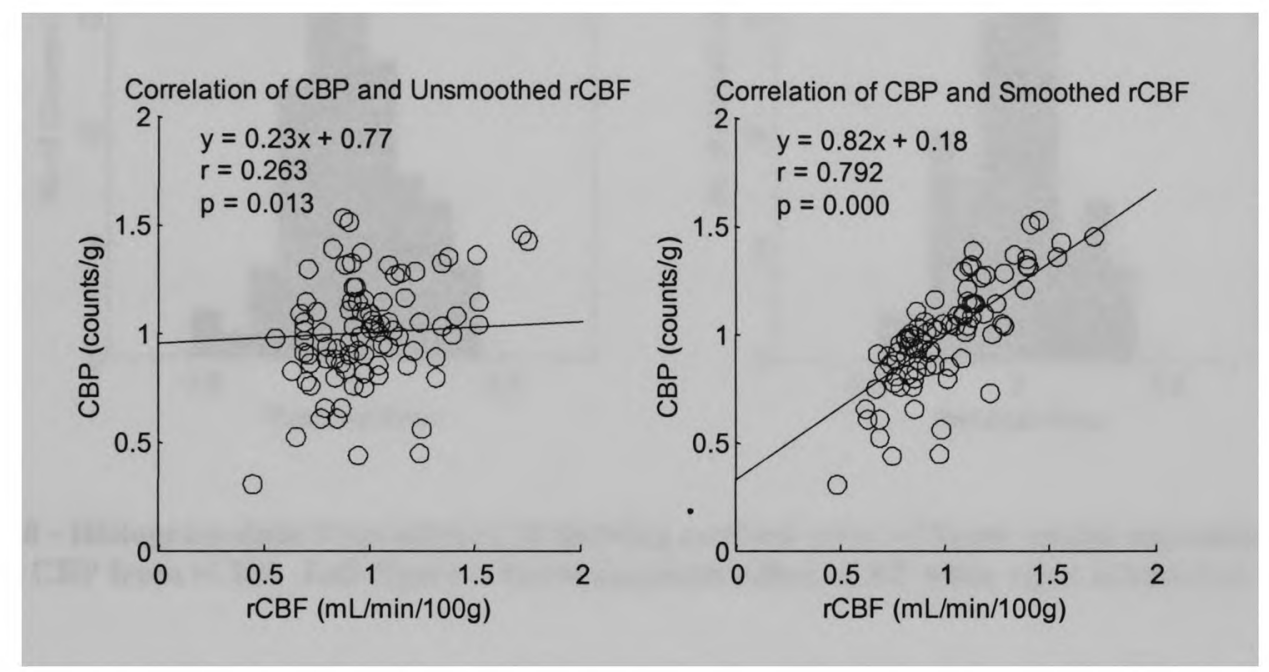

Figure 7 - Correlation data showing the effect of smoothing of the rCBF data for subject 10. Smoothing accounts for a large portion of the major source of experimental error introduced by imaging artifacts and to a lesser degree by erroneous reconstruction and misregistration.

The residuals for each subject were also examined for normality, the results for subject 10 can be seen in Figure 8.

If the residuals for all subjects are aggregated on the same histogram plot (Figure 9) the normal pattern holds. The narrower base of the smoothed data residuals histogram implies a more precise method of measurement. 

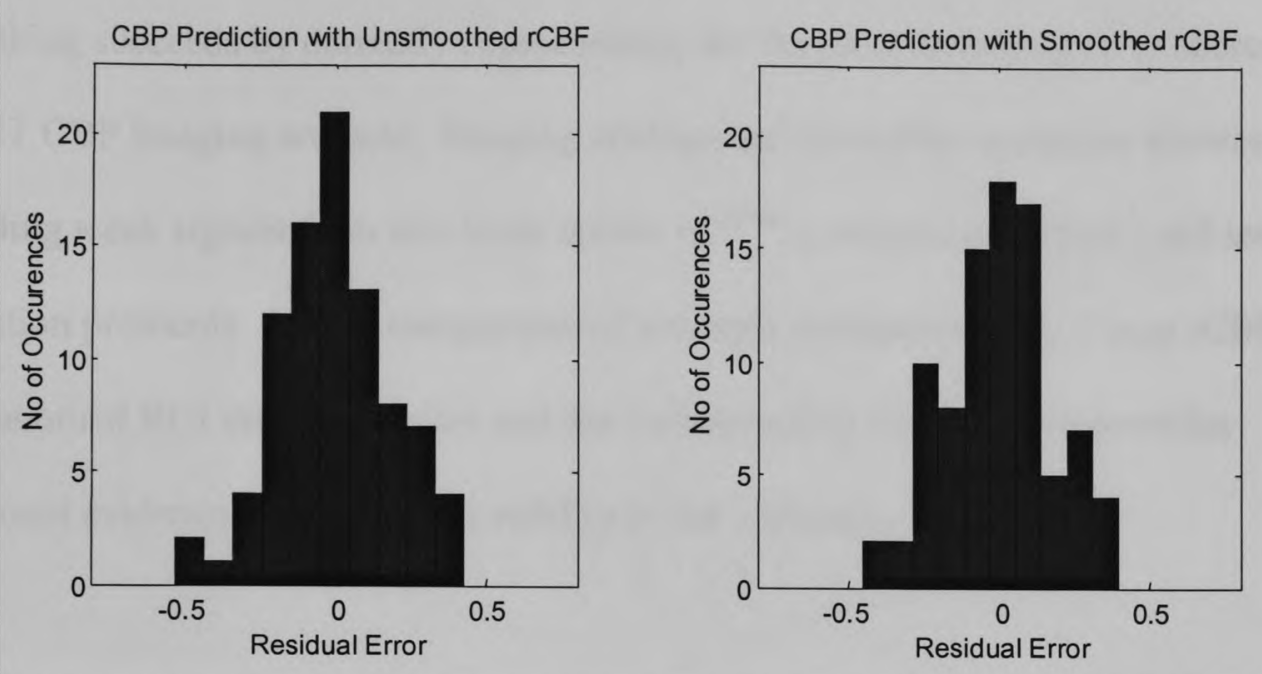

Figure 8 - Histogram data from subject 10 showing residual error of linear model regression used to predict CBP from rCBF. Left figure is based on unsmoothed $\mathrm{rCBF}$ while right is based on smoothed rCBF

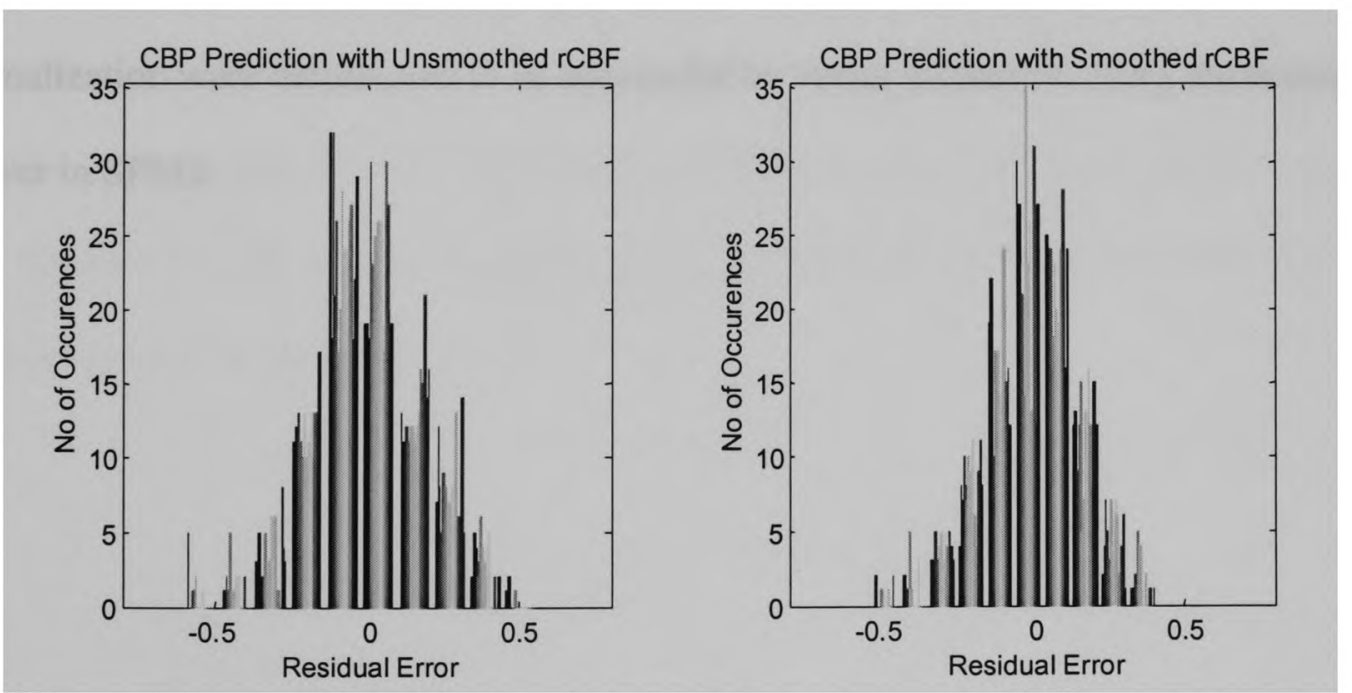

Figure 9 - Histogram data from all subjects combined showing the residual error of linear model regression used to predict CBP from rCBF. Left figure is based on unsmoothed $\mathrm{rCBF}$ while right is based on smoothed $\mathrm{rCBF}$ 
Recalling the major sources of error discussed earlier, it appears as though the $\mathrm{rCBF}$ smoothing succeeds by correctly compensating for the most damaging error source, SPECT CBP imaging artifacts. Imaging artifacts are caused by numerous sources including weak signals from low brain uptake of ${ }^{99 \mathrm{~m}} \mathrm{Tc}$, camera insensitivy and lowresolution protocols. Visual comparison of a sample reconstructed ROI map rCBF slice, the smoothed ROI map rCBF slice and the corresponding SPECT slice provides additional evidence supporting the validity of the approach.

\subsection{SPM}

Image data preprocessing consisted of spatial normalization between all subjects with subject 17 used as a template for registration. All matching between the template and subject stopped when the 0.05 error threshold was met. Results of spatial normalization were determined to be successful by visual inspection using the orthogonal viewer in SPM2. 

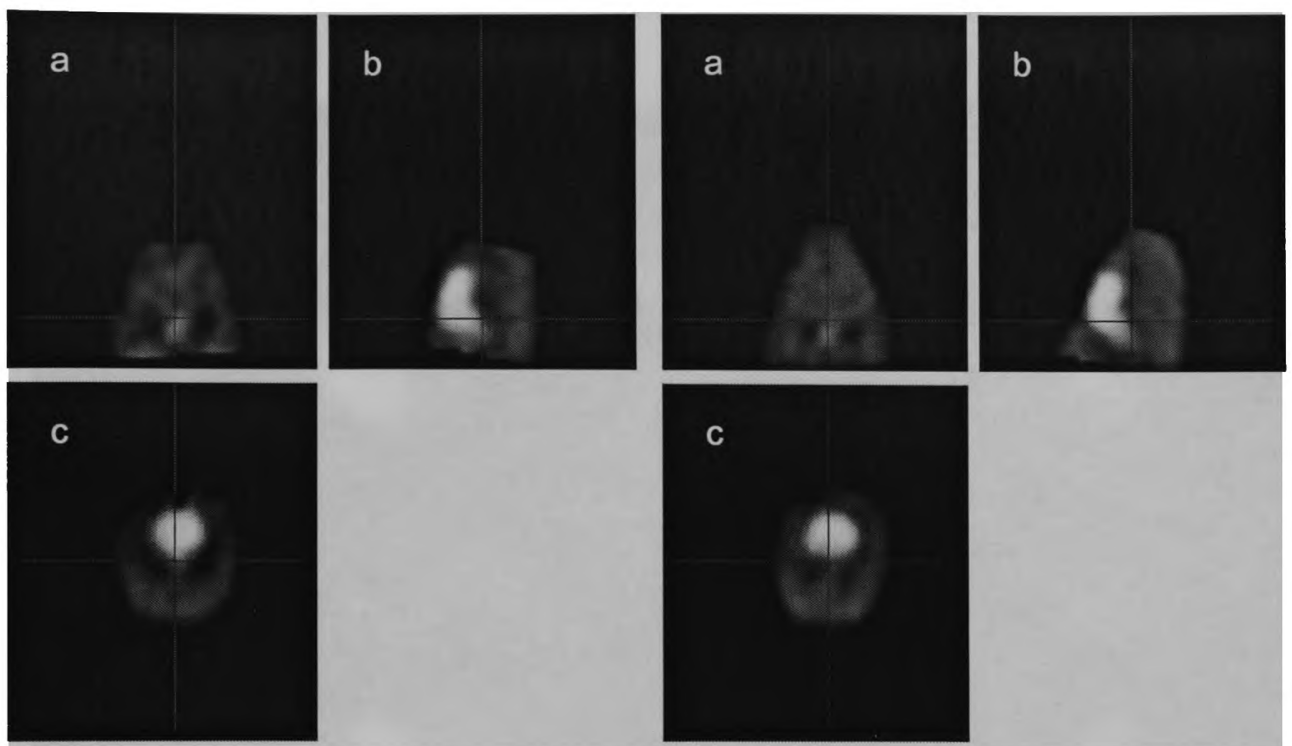

Figure 10 - Visual validation of spatial registration results was accomplished through side by side visual inspection using the SPM2 orthogonal viewer. Orthogonal views are (a) coronal (b) sagittal and (c) transverse. On the left side are the othrogonal views for subject 16 . On the right side are the three orthogonal views for the template, subject 17 . The brain is shown as the area of highest intensity in views (b) and (c).

Model specification and parameter estimation in SPM2 resulted in a map of pvalues for interpretation. The thresholded $\mathrm{p}$-value image in Figure 11 is given by the $\mathrm{t}$ contrast [ $\left[\begin{array}{ll}-1 & 1\end{array}\right]$ testing for any statistically significant decreases in CBP in the test group. The decrease of CBP in the left parietal region is clearly shown and is the only significant change reported in the brain area. 


\section{Inferior}

\section{Superior}

\section{Coronal}

Left

\section{Right}

\section{Sagittal}

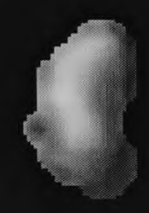

Figure 11 - Maximum intensity projections of significant decreases in SPECT CBP are rendered against a solid representation of the piglet brain. The location of the focal decrease in SPECT CBP is consistent with the left parietal injury treatment.

The exact location of the lesion in the left parietal lobe is given by the voxel coordinates in Table 4. Lesion size was calculated by multiplying the number of pixels, 11 , by the size of each pixel, $0.084 \mathrm{ccm} /$ pixel, yielding $0.924 \mathrm{ccm}$. 


\begin{tabular}{lllll}
\hline Number & $\mathrm{x}$ & $\mathrm{y}$ & $\mathrm{z}$ & $\mathrm{p}$ \\
\hline 1 & 32 & 39 & 11 & 0.0081 \\
2 & 33 & 39 & 11 & 0.0052 \\
3 & 34 & 39 & 11 & 0.0062 \\
4 & 32 & 39 & 12 & 0.0028 \\
5 & 33 & 39 & 12 & 0.0019 \\
6 & 34 & 39 & 12 & 0.0070 \\
7 & 32 & 40 & 12 & 0.0036 \\
8 & 33 & 40 & 12 & 0.0039 \\
9 & 33 & 39 & 13 & 0.0076 \\
10 & 32 & 40 & 13 & 0.0037 \\
11 & 33 & 40 & 13 & 0.0061 \\
\hline
\end{tabular}

Table 4 - Location and p-values for significant voxels in the lesion reported by SPM for control versus test group. Each significant voxel is based on control versus test comparison and was considered by the statistical test independently of other voxels in the brain area.

\subsection{SPM Validation using RMT Data}

Quantitative changes in rCBF were analyzed at each ROI using a one-way ANCOVA with two treatments, injury (test) or no injury (control). Results are outlined in Table 4 using a right hemisphere mean rCBF predictor variable. 


\begin{tabular}{|c|c|c|c|c|c|}
\hline \multicolumn{3}{|c|}{$\begin{array}{l}\text { Right Hemisphere } \\
\text { Mean rCBF } \\
\text { Predictor } \\
\end{array}$} & \multirow[b]{2}{*}{$\begin{array}{c}\text { Control } \\
\text { Mean rCBF } \\
(\mathrm{mL} / 100 \mathrm{~g} / \mathrm{min})\end{array}$} & \multirow[b]{2}{*}{$\begin{array}{c}\text { Test } \\
\text { Mean rCBF } \\
(\mathrm{mL} / 100 \mathrm{~g} / \mathrm{min})\end{array}$} & \multirow[b]{2}{*}{$\begin{array}{l}\text { Percent } \\
\text { Difference } \\
\text { rCBF }\end{array}$} \\
\hline ROI & $P(F \leq F p)$ & $\begin{array}{l}\text { Adjusted } \\
\mathrm{p}\end{array}$ & & & \\
\hline 3 & 0.002 & 0.158 & 93.38 & 69.43 & - \\
\hline $8 \mathrm{~L}$ & 0.005 & 0.431 & 70.89 & 53.01 & - \\
\hline 11 & 0.000 & 0.009 & 91.93 & 62.34 & -32.2 \\
\hline $12 \mathrm{~L}$ & 0.003 & 0.282 & 120.50 & 90.52 & - \\
\hline 24 & 0.004 & 0.317 & 74.69 & 89.16 & 19.4 \\
\hline $26 \mathrm{~L}$ & 0.003 & 0.290 & 94.41 & 51.86 & -45.1 \\
\hline 36 & 0.009 & 0.748 & 94.01 & 118.26 & 25.8 \\
\hline 33L & 0.000 & 0.000 & 102.25 & 31.17 & -69.5 \\
\hline $34 \mathrm{~L}$ & 0.002 & 0.141 & 81.54 & 50.72 & -37.8 \\
\hline $39 \mathrm{~L}$ & 0.000 & 0.009 & 110.89 & 50.26 & -54.7 \\
\hline $40 \mathrm{~L}$ & 0.000 & 0.000 & 98.01 & 68.11 & -30.5 \\
\hline
\end{tabular}

Table 5 - RMT rCBF mapping results. Left hemisphere ROIs have L suffix, while right hemisphere ROIs have no suffix. F statistic is used to derive p-values. Adjusted p-values are based on Bonferroni correction.

ROIs with a significance of $(\mathrm{p}<0.01)$ are shown as an informal compensation method for multiple comparisons. The adjusted $\mathrm{p}$ column uses Bonferroni correction and results in p-values that may be overadjusted [3]. Similar to the SPM results, rCBF data showed a significant decrease in the left parietal region $(26 \mathrm{~L}, 33 \mathrm{~L}, 34 \mathrm{~L}, 39 \mathrm{~L}, 40 \mathrm{~L})$. Interestingly, an effect that was not present in the SPM analysis, but present here, was a significant $25 \%$ increase in the brain stem region (36). However, significance in this region was reached only for the uncorrected p-value. Additional study may be able to tell whether the effect is real or a false positive. 


\section{Discussion}

SPECT imaging is an important tool in assessing TBI [1,31]. Animal based pediatric models of TBI are especially suited for studying specific $\mathrm{rCBF}$ responses to TBI by controlling the magnitude and location of the injury [18]. Functional data in animal model experiments is often validated using the gold standard RMT method which poses formidable visualization and registration challenges $[7,41]$. These challenges were overcome in this study through systematic application of a set of techniques.

In order to visualize the ROI maps they were traced and reconstructed similarly to Gross et al [13] using a raster scanning algorithm proposed by Heckbert [34]. Registration of the ROI maps to the functional SPECT data did not require automated block matching algorithms [20] because only nine slices per subject required registration. The visualization and registration process as a whole complements similar work such as registration of PET and ex vivo autoradiography data using manual matching of ROIs between modalities [42], registration of VOIs marked on digitized serial slice images and PET data using a maximization of mutual information metric between image pairs [43], and registration of serial slices to MRI by manipulating the position of the brain during sectioning [44]. No study has been found dealing specifically with the registration of anatomical RMT derived ROI maps and nuclear medicine images. 
Characterization of lesions induced by the TBI has been addressed in both animal models and in retrospective studies in humans [8]. SPM is becoming an increasingly popular approach in the field of statistical neuroimaging for interpreting lesions. Similar to clinical functional brain studies of head injury $[11,23]$, SPM analysis was performed to detect and characterize CBP at the precision afforded by the SPECT camera and image volume reconstruction configurations.

Although detection by SPM of the lesion induced through FPI was not surprising, valuable information was gained as to its spatial extent and relative magnitude. The major drawback of the SPM approach to lesion detection is the requirement of multiple subjects for each treatment (i.e. injury) in order to find statistically significant areas of the image (e.g. a lesion). If the method were to be used in a clinical setting additional research would need to be done on how to achieve statistical power using a single subject. One approach might consider comparing multiple scans of a single patient versus a reusable control group.

Characteristics of the lesion could be used to help build automated algorithms for the detection of TBI in pediatric patients. Existing algorithms designed to detect SPECT CBP asymmetries in a single adult subject use statistical techniques coupled with threshold comparison between the two hemispheres of the brain [5]. These algorithms are generally successful at finding asymmetries in the brain. However, whether the lesion is pathological in nature must be further studied by a clinician. Combining 
knowledge of the characteristics of this lesion from the study with automated searching could lead to a more powerful and useful medical image processing algorithm.

Clinical applications notwithstanding, the SPM methodology as implemented here is unique in that no other studies using SPM to study functional data in an animal model were found to have been reported.

The reconstruction, registration and smoothing techniques used to create the aggregate CBP-rCBF linear regression models can also be used for fluorescent microspheres studies such as by the one by Epstein et al. [19] and for arbitrary specimens, or organs. Future work needs to be done to automate the reconstruction and registration processes. It is anticipated that the tools developed here can be useful to other groups performing TBI research in animal models, or even more generally in research requiring map definition, registration and analysis. 


\section{Conclusion}

The original objectives of this work were to study the suitability of the application of SPM to an animal model of TBI and the correlation between the SPECT CBP and reconstructed RMT rCBF data.

The hypothesis of SPM suitability for application to the experimental model and ability to providing insight into $\mathrm{CBF}$ in response to traumatic injury was validated by the SPECT SPM result of a decrease in CBP at the left parietal region injury area of the test group. Correction of the global activity using a right hemisphere average predictor was critical to achieving this result. A more sophisticated model might take into account additional predictor covariates individually instead of in a general manner, as with the right hemisphere predictor. Additional covariates capable of being examined in this study were animal weight, the time between dose and injection and the total injected dose. It is anticipated that correction by the latter two methods would yield minimal improvement given their relative consistency between subjects.

Statistical mapping of the reference RMT rCBF data confirms the focal decrease found with SPECT and SPM. Multiple comparisons correction had a large effect on reported p-values for the RMT data and for this reason both adjusted and non-adjusted pvalues were presented. For example, the increase in $\mathrm{rCBF}$ in brain stem region 36, an expected effect of injury, becomes insignificant with Bonferroni correction. Further review or study is required into the possible physiological significance of this effect. 
Regarding the hypothesis of clinical SPECT CBP correlation with RMT rCBF, the aggregate linear regression model of $\mathrm{CBP}$ using $\mathrm{rCBF}$ was not as strong as expected due to SPECT imaging artifacts and the relatively small size of the piglet brain. Nonetheless, the model is useful for validation of the SPECT data. Advancements in clinical SPECT imaging such as greater sensitivity, resolution and attenuation correction, or use of a smaller detector tailored to animal research, would undoubtedly lead to improved accuracy and detection by minimizing artifacts such as partial volume effects and photon scatter while increasing sensitivity. 